\title{
A Geophysical Review of the Seabed Methane Seepage Features and Their Relationship with Gas Hydrate Systems
}

\author{
Jinxiu Yang $\mathbb{D}^{1,2}$ Mingyue Lu, ${ }^{2}$ Zhiguang Yao, ${ }^{3,4}$ Min Wang, ${ }^{1,2}$ Shuangfang Lu, ${ }^{1,2}$ Ning Qi, ${ }^{2}$ \\ and Ying $\mathrm{Xia}^{2}$ \\ ${ }^{1}$ Key Laboratory of Deep Oil and Gas, China University of Petroleum (East China), Qingdao 266580, China \\ ${ }^{2}$ School of Geosciences, China University of Petroleum (East China), Qingdao 266580, China \\ ${ }^{3}$ Engineering Technology Research Company Limited, China National Petroleum Corporation, Tianjin 300451, China \\ ${ }^{4}$ Key Laboratory of Offshore Engineering, China National Petroleum Corporation, Tianjin 300451, China
}

Correspondence should be addressed to Jinxiu Yang; yangjinxiu@upc.edu.cn

Received 12 March 2021; Revised 8 July 2021; Accepted 6 September 2021; Published 12 October 2021

Academic Editor: Wei Zhang

Copyright (c) 2021 Jinxiu Yang et al. This is an open access article distributed under the Creative Commons Attribution License, which permits unrestricted use, distribution, and reproduction in any medium, provided the original work is properly cited.

Seabed methane seepage has gained attention from all over the world in recent years as an important source of greenhouse gas emission, and gas hydrates are also regarded as a key factor affecting climate change or even global warming due to their shallow burial and poor stability. However, the relationship between seabed methane seepage and gas hydrate systems is not clear although they often coexist in continental margins. It is of significance to clarify their relationship and better understand the contribution of gas hydrate systems or the deeper hydrocarbon reservoirs for methane flux leaking to the seawater or even the atmosphere by natural seepages at the seabed. In this paper, a geophysical examination of the global seabed methane seepage events has been conducted, and nearby gas hydrate stability zone and relevant fluid migration pathways have been interpreted or modelled using seismic data, multibeam data, or underwater photos. Results show that seabed methane seepage sites are often manifested as methane flares, pockmarks, deep-water corals, authigenic carbonates, and gas hydrate pingoes at the seabed, most of which are closely related to vertical fluid migration structures like faults, gas chimneys, mud volcanoes, and unconformity surfaces or are located in the landward limit of gas hydrate stability zone (LLGHSZ) where hydrate dissociation may have released a great volume of methane. Based on a comprehensive analysis of these features, three major types of seabed methane seepage are classified according to their spatial relationship with the location of LLGHSZ, deeper than the LLGHSZ (A), around the LLGHSZ (B), and shallower than LLGHSZ (C). These three seabed methane seepage types can be further divided into five subtypes considering whether the gas source of seabed methane seepage is from the gas hydrate systems or not. We propose subtype B2 represents the most important seabed methane seepage type due to the high density of seepage sites and large volume of released methane from massive focused vigorous methane seepage sites around the LLGHSZ. Based on the classification result of this research, more measures should be taken for subtype B2 seabed methane seepage to predict or even prevent ocean warming or climate change.

\section{Introduction}

In recent years, seabed methane seepage has gained attention from all over the world as an important source of greenhouse gas emission which may affect climate change or even global warming (Figure 1) [1-4]. Seabed methane seepage features, also termed as "cold seeps," refer to the seeping or venting features of fluids at the seabed. Seeping often indicates slow fluid fluxes, venting indicates fast vigorous fluxes, and fluids involve hydrocarbon gas especially methane, water, and even sediments [1]. Seabed seepage features are often manifested as methane flares, pockmarks, deep-water corals, authigenic carbonate, and gas hydrate pingoes at the seabed, which are often related to vertical or subvertical fluid migration conduits $[1,5]$. According to Talukder [1], there are three elements for seabed methane seepage including source, plumbing system, and seabed methane seepage structures. Seabed methane seepage is commonly used as 


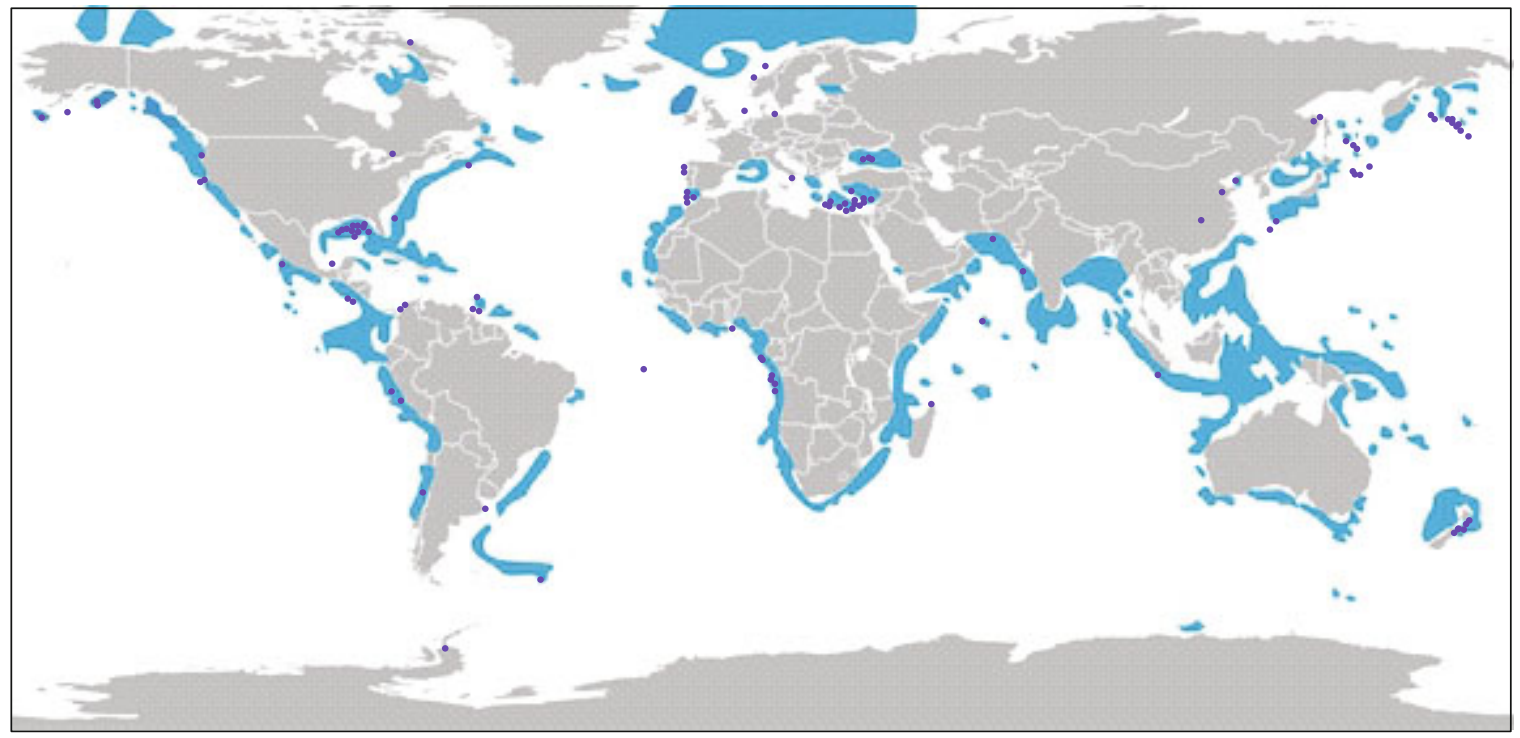

Gas hydrate

Cold seeps

(a)

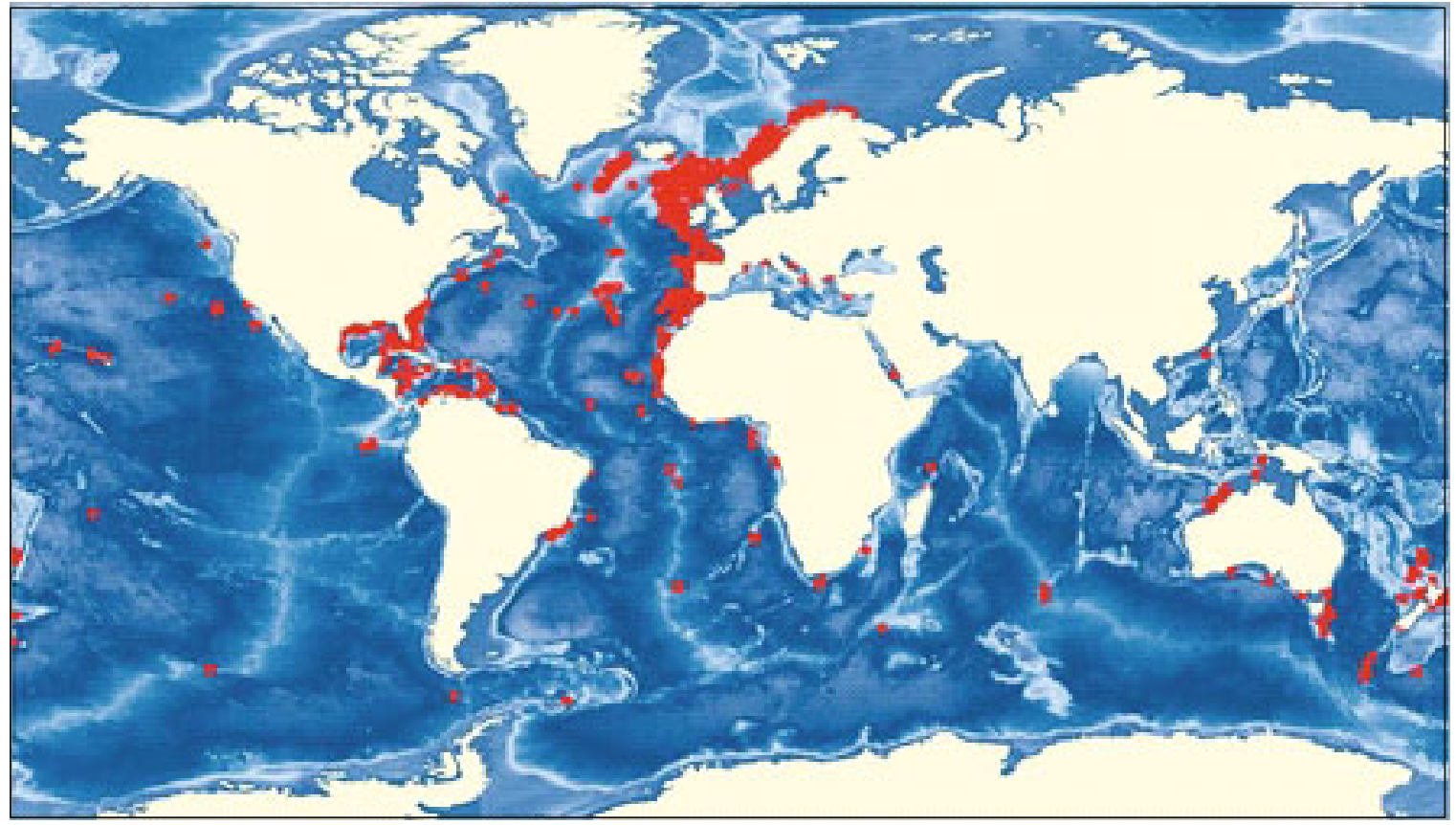

Coral reef

(b)

FIGURE 1: (a) Global distribution of marine gas hydrates and cold seeps (modified from Klauda and Sandler [6]; German et al. [7]). (b) Global distribution of deep-water reef-forming corals (from Roberts et al. [8]).

an indicator of subsurface gas reservoirs or gas hydrate systems, which presents the leakage of methane from the shallow lithosphere to the hydrosphere and atmosphere. However, most case studies on seabed methane seepage at present often focus on description of the seepage features, and only a few studies investigated the source of methane and the migration history from the source to the seabed seepage sites.
Gas hydrate systems are often regarded as an important methane sink because of the large amounts of methane they captured, often occurring in shallow sediments at water depths greater than a few hundred meters, and the base of gas hydrate stability zone varies in different research areas with different latitudes controlled by the pressure and temperature conditions (Figure 1(a)) [9, 10]. Gas hydrates are sensitive to environmental variations like sea level change, 
seabed temperature change (also called ocean warming), seabed bottom currents, salinity change of seawater, and ice sheet fluctuations, all of which can change the pressure and temperature conditions of gas hydrate stability, therefore causing gas hydrate dissociation and probably subsequent methane seepage at the seabed [3, 4, 11-15]. For example, NASA scientists observed millions of seabed methane seeps in the Arctic region and it was proposed that the postglacial climate warming and deglaciation caused the dissociation of gas hydrates, therefore leading to the seabed methane release $[16,17]$. Similar phenomena have also been observed in US Atlantic margin [18-20], offshore Svalbard [21-23], and Spitsbergen continental margin [21], where the seabed methane seepage features are all interpreted to be due to the dissociation of gas hydrates.

However, not all seabed methane seepage features are related to gas hydrate systems and some may be from the deeper gas reservoirs [24]. Due to the proper temperature and pressure conditions for gas hydrate formation at the seabed, gas hydrate outcrops are often observed at the seabed methane seepage sites, but this does not mean the gas source is directly from the gas hydrate systems. Therefore, it is important to analyse the gas source of seabed methane seepage and its relationship with the gas hydrate systems, which can help better understand the potential contribution of gas hydrate systems or the deeper gas reservoirs for methane leaking to the seawater or even the atmosphere. In this paper, we investigate the seabed methane seepage case studies all over the world, the relevant fluid migration pathways and gas source are analysed, and its relationship with the gas hydrate systems is used to classify the types of seabed methane seepage.

\section{Geological Conditions}

2.1. Seabed Methane Seepage. Seabed methane seepage represents the transfer of methane from the geosphere to hydrosphere, biosphere, or even atmosphere, which is regarded as a process of the Earth's carbon recycling [25, 26]. Seabed methane seepage features can occur globally at different geological settings including active margins (e.g., convergent margin, accretionary margin, erosive margins, and transform margins) and passive margins $[25,27]$. Seepage fluid composition often includes water, free gas (especially methane), and some sediments, and they transfer from different sources upwards to the seafloor by different forcing mechanisms such as sediment compaction, methane overpressure caused by gas hydrate dissociation, biogeochemical reactions, biological activities, overpressure, and facies changes $[25,26,28]$. Apart from seabed seepage manifestations (e.g., pockmarks, deep-water corals, and authigenic carbonate crusts on mounds or pavements), fluid conduit features (such as slope failures, faults, mud volcanoes, scars, scarps, and bulges) also play a big role in facilitating fluid escape and seabed methane seepage formation $[29,30]$. The magnitude of seabed methane seepage is difficult to determine because the seepage features, including bubble size, velocity, and concentration, often show significant temporal variability on time scales from seconds to decades $[31,32]$. How- ever, seabed manifestations and related fluid conduits provide clues for methane source information, such as the source depth, its relationship with the gas hydrate systems, and possible roles in material cycling, which is the objective of this study.

In this paper, we mainly focus on the methane source of the seabed methane seepage and discuss its relationship with the gas hydrate systems by analysing their spatial relationship. Global case studies from Niger Delta, offshore Mauritania, and South China Sea and other multilatitude regions are discussed in this paper, which are featured by different geological conditions. For example, Niger Delta is located on the passive continental margin on the west coast of central Africa, and the structural units of the Niger Delta can be divided into three domains, the extension domain, the translation domain, and the compression domain, featured by growth faults, mud diapirs, shale diapirs, and foldingthrusting features $[33,34]$. Offshore Mauritania is located on the West African passive continental margin, with limited faulting and fracturing development $[10,35,36]$. The South China Sea is a large marginal sea characterized by continental rifting and seafloor spreading, with high heat flow value [37, 38]. Additionally, large-scale gas chimneys are common in South China Sea with faults and fractures existing in the interior of the gas chimneys. In these offshore study areas, gas hydrate systems are often developed, with focused fluid migration providing gas source for hydrate formation which may also have caused the seabed methane seepage features.

2.2. Gas Hydrate Stability Zone (GHSZ). Marine gas hydrates occur worldwide on continental active and passive margins, marginal seas, and island slopes, and the gas hydrate stability zone (GHSZ) is mainly determined by factors including seabed temperature, seabed depth, geothermal gradient, gas composition, and pore water salinity, with temperature and pressure being the primary factors affecting the phase equilibrium of gas hydrates [20,39,40]. Gas hydrates play dual roles in upward methane migration during geological periods, which can act as time-variable sources or sinks for methane $[41,42]$. On the one hand, methane can be captured in the GHSZ by forming gas hydrates during upward migration. On the other hand, hydrate dissociation will release a great volume of methane which escapes to the shallower sediments or even the seawater by seabed methane seepage.

In seismic data, the base of GHSZ is often indicated by bottom simulating reflection (BSR), which is normally characterized by strong amplitude, negative polarity, and crosscutting chronostratigraphic reflections. In high-resolution seismic data, BSR is often featured as aligned amplitude terminations [43-45]. Due to the shallow burial and weak stability of the gas hydrate system, the gas hydrate stability conditions are easy to be affected by ocean currents, sedimentation, diapirism, and other environmental changes, resulting in the formation and decomposition of natural gas hydrates [46-48]. The methane captured and stored in the gas hydrate systems is therefore prone to escape to the seawater or even atmosphere through seabed seepage 
features. As a result, it is necessary to better understand the spatial relationship between seabed methane seepage and the gas hydrate systems, as well as related fluid migration conduits.

However, the gas hydrate occurrence is not always indicated by an obvious BSR in seismic data, and gas hydrates may exist without BSR such as in Site 994 of ODP Leg 164 [10]. When it exists, the BSR often decreases as the seabed shallows until it intersects with the seabed, where it is known as the landward limit of gas hydrate stability zone (LLGHSZ) representing the shallowest water depth of GHSZ. Due to limited methane supply and the anaerobic oxidation of methane (AOM) at shallow depth, both BSR and LLGHSZ are rarely visible in the vicinity of the theoretical LLGHSZ in seismic data in many gas hydrate occurrence areas [4, $35,48-50]$. In areas without BSR development, it is difficult to recognize the GHSZ which may hinder judging whether the methane source for the seabed methane seepage is related with the gas hydrate systems or not. In these circumstances, the BSR can be numerically modelled in MATLAB software by assuming a proper gas composition and a reasonable salinity for the modelled gas hydrate systems (Figure 2). The temperature distribution is calculated by a two-dimensional steady-state heat conduction model, with related equation discretized in space using finite differences and solved directly using MATLAB's MLDIVIDE function. The upper boundary condition is determined by fixing the temperature at the seabed to the seawater temperature at the depth of the local seabed surface (retrieved from the World Ocean Database; Figure 2). The lower boundary condition is applied by fixing the geothermal gradient to a uniform value at $5 \mathrm{~km}$ below the seabed. This boundary geothermal gradient is therefore referred to as the regional geothermal gradient (RGG). The depth of the seabed is calculated from the TWT time, which is converted to depth using a water velocity of $1.5 \mathrm{~km} \mathrm{~s}^{-1}$. A pressure profile is obtained by assuming a constant hydrostatic pressure gradient of $10.09 \mathrm{MPa} \mathrm{km}^{-1}$. Then, the depth of the BSR can be determined using the hydrate stability curve which is suitable for the studied gas hydrate system.

For example, the gas hydrate stability curve for pure methane with a salinity of $35 \mathrm{wt}$. \% given by Moridis [51] is used to predict the BSR locations in two seismic profiles from Niger Delta and Qiongdongnan Basin of South China Sea (Figure 3). The depth of the BSR is determined by hydrate stability conditions and can be calculated by numerical modelling through assuming proper controlling parameters. Regional geothermal gradient (RGG) is considered as a calibration parameter in this context, and an optimal fit between the modelled and observed BSR was obtained by adjusting the RGG value in the geothermal gradient range of the study area. Results show that optimal fit between the modelled and observed BSR can be obtained by setting a RGG value of $4.2^{\circ} \mathrm{C} / \mathrm{hm}$ for the seismic profile from Niger Delta and by setting a RGG value of $3.5^{\circ} \mathrm{C} / \mathrm{hm}$ for the seismic profile from Qiongdongnan Basin (Figure 3). For both seismic profiles, the BSRs at the crest of the anticlines (Figure 3(a)) or above the focused cluster of faults (Figure 3(b)) are vaulted, probably due to the thermal anom- alies transported from underneath by folding or faulting. The results are dependent on several assumptions including velocities of seawater and sediments, as well as gas composition. Changes in these parameters would lead to different RGG values, which represent the uncertainties of this modelling. With the numerical modelling, the GHSZ range can be predicted which can help characterize the spatial relationship between gas hydrate systems and the seabed methane seepage features, therefore evaluating the role of gas hydrate systems in forming seabed methane seepage.

\section{Characterization of Seabed Methane Seepage Manifestations}

Seabed methane seepage is often characterized by seabed manifestations such as methane flares, pockmarks, carbonate crusts on mounds or pavements, coral reefs, mud volcanoes, hydrate pingoes, and chemosynthetic biological communities $[1,4,12,52,53]$. The size and density of these seabed manifestations reflect the volume of methane transferred from geosphere to hydrosphere or even the atmosphere, whose presence also indicates the existence of underlying hydrocarbon reservoirs or gas hydrate systems $[1,53]$. The gas source of methane seepage at the seabed can be from shallow biogenic gas and deep thermogenic gas or from gas hydrate dissociation [25]. However, gas hydrate outcrops are often observed at the seabed methane seepage sites regardless of their gas sources, which are mainly determined by the proper temperature and pressure conditions at the seabed. Therefore, we propose that the presence of gas hydrate outcrops does not indicate that the gas source of seabed methane seepage is directly from the gas hydrate systems. In this section, the detailed characteristics of main seabed methane seepage features are introduced, including pockmarks and carbonates, deep-water corals, and mud volcanoes. Related fluid migration conduits are interpreted, as well as nearby gas hydrate systems. Based on a combined analysis of these factors, the specific gas source of seabed methane seepage can be determined which help accurately evaluate the role of different gas sources in affecting the carbon cycling by seabed methane seepage.

3.1. Pockmarks and Carbonates. Pockmarks are crater-like depressions developed in fine marine or lacustrine sediments in various geological environments, probably due to overpressure discharge of subsurface fluids at the seabed [54-56]. As the result of fluid migration through sediments and expulsion at the seabed, pockmarks were often interpreted to overlie hydrocarbon reservoirs or gas hydrate systems (including gas hydrates and underlying free gas zone) which are the possible fluid sources [1,53]. Limited data has been known about the process of fluid venting which formed and maintained the pockmarks, but several mechanisms have been raised as drivers of fluid migration including migration from overpressured hydrocarbon reservoirs along vertical conduits like faults, fractures, and gas chimneys, sediment compaction, overpressure dissipation via focused vertical fluid migration conduits or salt/mud diapirs, gas hydrate dissociation, and related methane seepage 

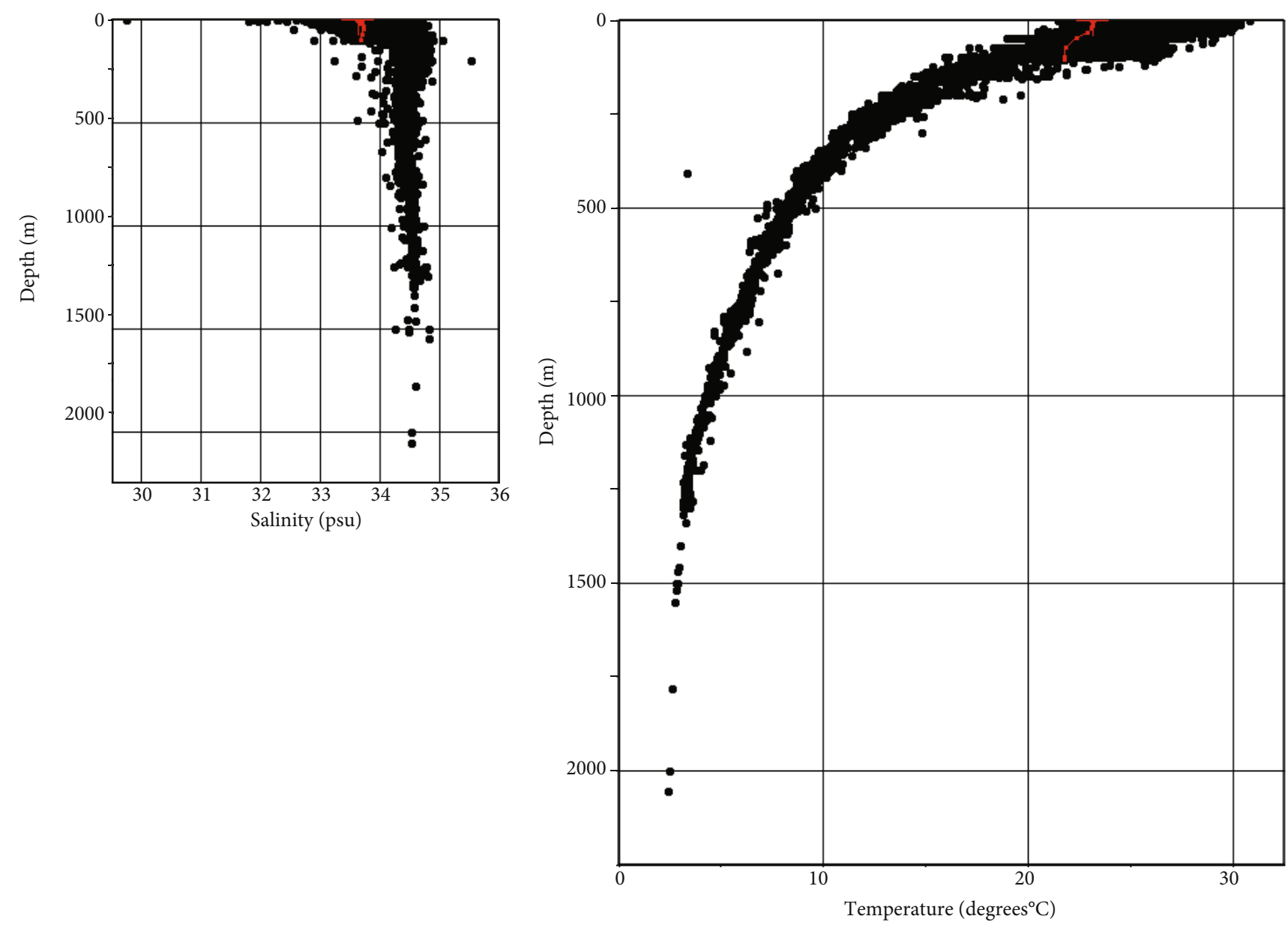

(a)

(b)

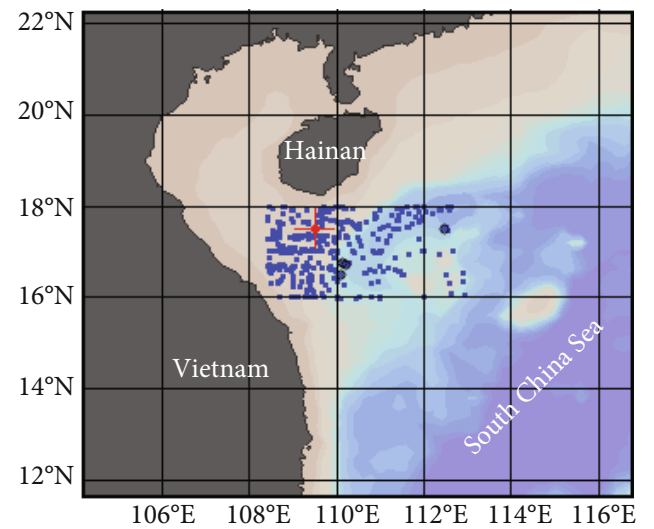

(c)

FIGURE 2: Data used in numerical modelling of the BSR. (a) Scatter plot of the sea water salinity with varying seabed depths in the northern South China Sea. (b) Scatter plot of the sea water temperature with varying seabed depths in the northern South China Sea. (c) Location map of the data points.

around the LLGHSZ. Pockmarks occur in both random and nonrandom distribution patterns, probably controlled by both the distribution of subsurface fluid conduits and the overpressured fluid source [53]. However, at the LLGHSZ where gas hydrate dissociation often occurs, pockmarks may occur in linear patterns without obvious subsurface fluid migration conduits since the gas source is directly from in situ hydrate dissociation.

In global case studies, there are different kinds of pockmarks. For example, giant pockmarks formed in the Faroe Shetland Basin due to a sudden expulsion of a methane reservoir which is deeper than the GHSZ, probably driven by 


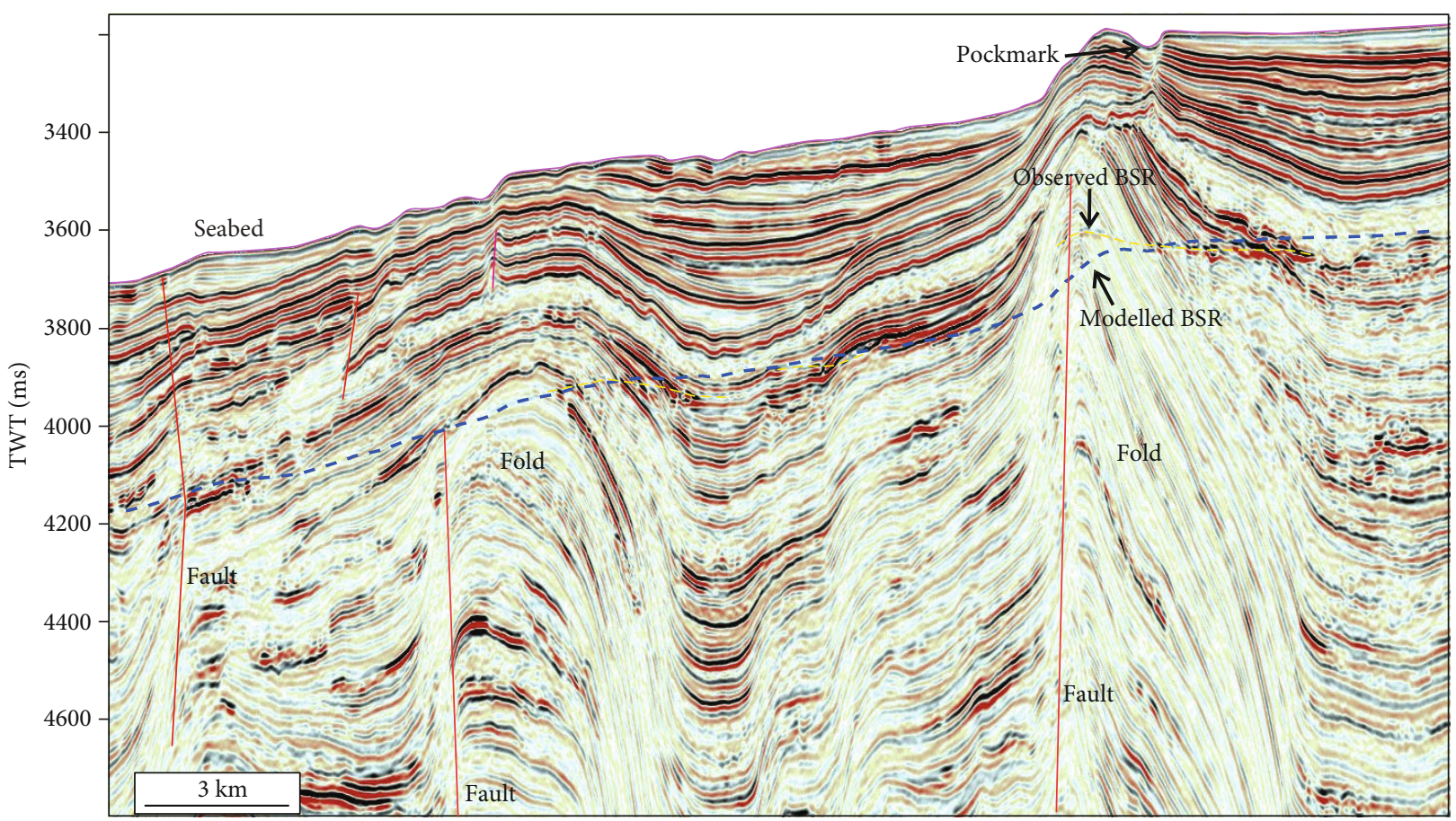

(a)

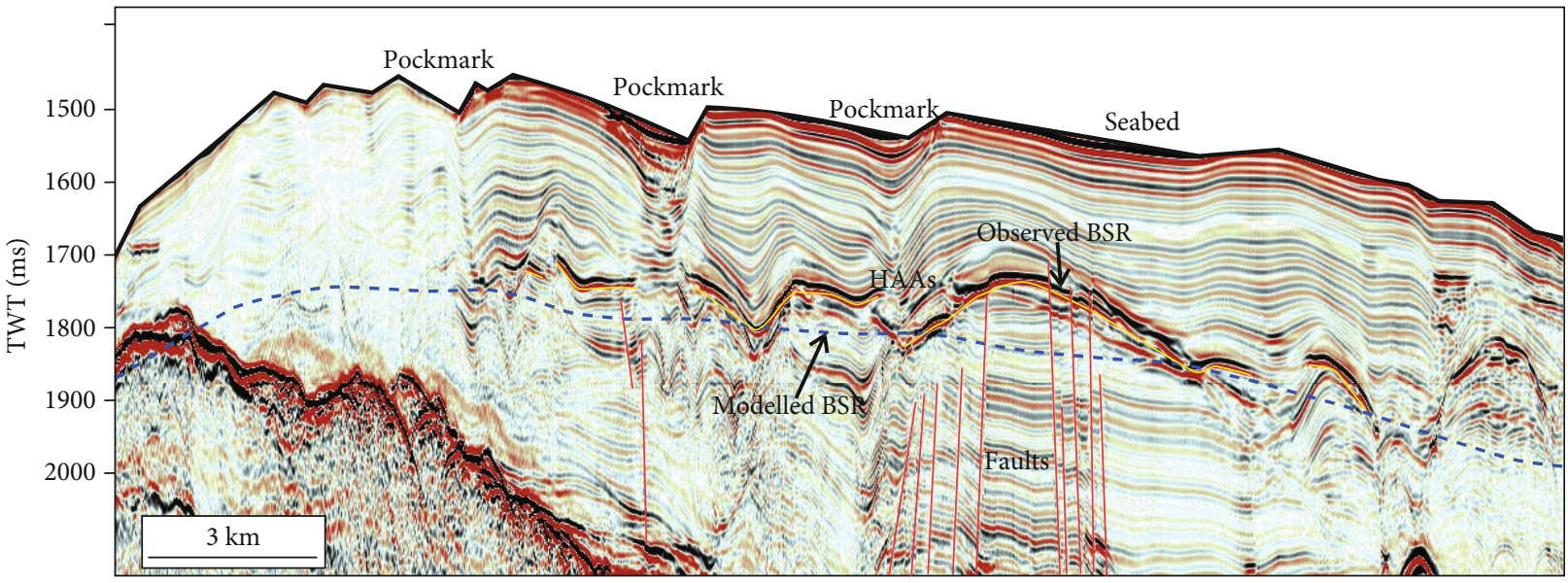

(b)

FIGURE 3: Numerical modelling results showing the observed BSR (yellow line) and the modelled BSR (blue line). (a) A seismic profile from Niger Delta showing faults and seabed pockmark. (b) A seismic profile from Qiongdongnan Basin showing faults and seabed pockmarks.

natural reservoir overpressure [57]. The overpressure was interpreted to be mainly trigger by tectonic compression because other causes are too gradual and modest, which opened the vertical conduits by exploiting the weakness in the seal. Figure 4 shows the relationship between pockmarks and gas hydrate systems in the U.S. Atlantic continental margin, and there are two types of pockmarks considering their relationship with the gas hydrate systems [18, 19]. The pockmarks much shallower than the LLGHSZ are caused by the overpressure of the shallow gas reservoir, irrelevant to the gas hydrate systems (Figure 4(a)). In Figure 4(b), more than 5000 pockmarks were observed in a $1100 \mathrm{~km}$ stretch along the strike of the U.S. Atlantic continental margin, and most of the pockmarks are located around the LLGHSZ. Gas hydrate dissociation due to differential sediment loading and global warming was proposed to be responsible for the formation of these pockmarks, which caused pore fluid overpressure and vertical fluid migration [19]. Within the GHSZ scope, there are few pockmark occurrence at the seabed compared to the huge amount of pockmarks around the LLGHSZ. The fluid source for these pockmark formation could be from both gas hydrate systems and deeper hydrocarbon reservoirs, which can be deduced by the vertical distribution of fluid migration conduits $[58,59]$. As shown in Figure 4(c), these two pockmarks were formed as a result of overpressure dissipation, and the overpressure was probably caused by the continuous accumulation of free gas below the base of gas hydrate stability 


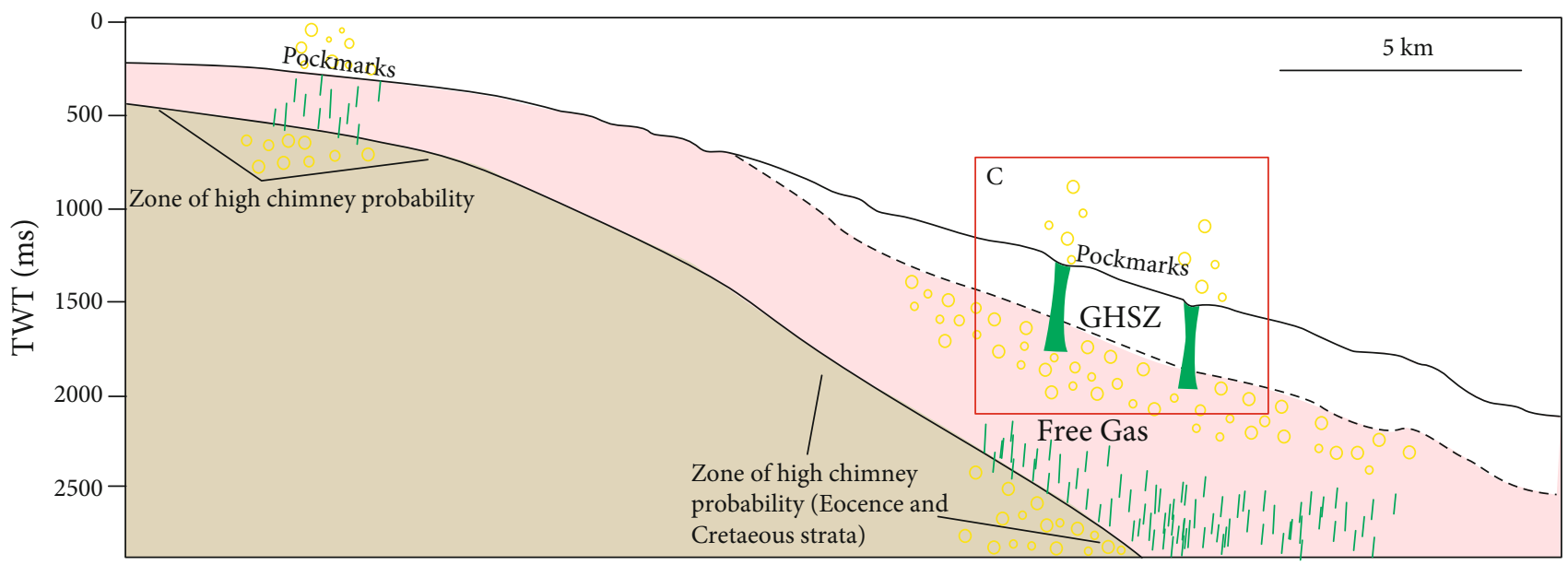

(a)

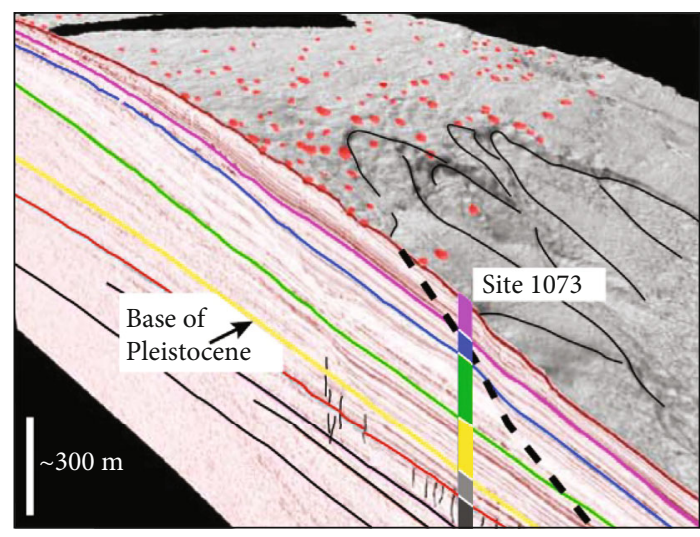

(b)

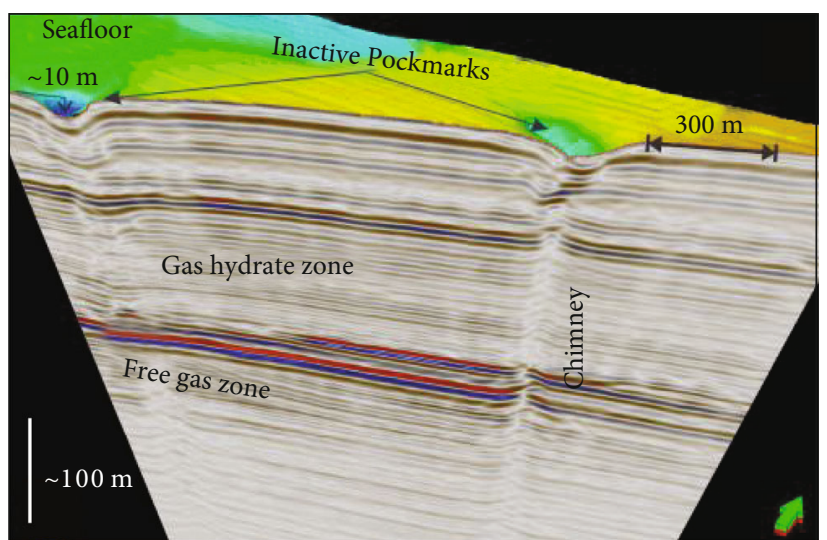

(c)

FIGURE 4: (a) Schematic diagram showing pockmarks, related gas chimneys, and nearby gas hydrate stability zone (modified from Brothers et al. [19]). Pockmarks in the updip of the GHSZ are interpreted to be due to the overpressure of the shallow gas reservoir, which is irrelevant to the gas hydrate system. Additionally, the pockmarks within the GHSZ scope were caused by the overpressure of the free gas zone which triggered the formation of gas chimneys and pockmarks. (b) 3D view of seismic data showing the landward limit of GHSZ and nearby numerous pockmarks which were the result of gas hydrate dissociation due to the seaward retreat of the GHSZ during global warming or sea level change (modified from Brothers et al. [19]). (c) Seismic data showing the inactive pockmarks, gas chimneys, and the free gas zone below the base of the gas hydrate stability zone (modified from Plaza-Faverola et al. [60]).

zone [60]. The pockmarks became inactive shortly after transient initial expulsion, which may be reactivated when the free gas accumulates again and generates required overpressure $[57,60]$. Besides the present pockmarks, there are also paleopockmarks which are buried pockmarks indicating the fluid escape features in the geological past [61] (Figures 5(c) and 5(d)).

Authigenic carbonates often extensively coexist with methane seepage features in different geological settings, representing the removal of methane and inhibiting its emission to the sea water or even the atmosphere [62-64]. It is commonly believed that the biological process of anaerobic oxidation of methane $(\mathrm{AOM})$ couple to sulphate reduction (SR) is mediated by anaerobic methanotrophs and sulphate-reducing bacteria in anaerobic environments, with authigenic carbonates being the main by-product of the AOM-SR process [63-66]. According to previous studies, the formation of carbonates will eventually seal the seeping feature, which is characterized by partial seepage clogged with sediments and bacterial mats first and complete blockage by carbonate precipitation which builds up a carbonate crust $[1,67]$. Additionally, authigenic carbonates are often regarded as associated with the gas hydrate systems [68, 69]. A case study from the southern summit of the Hydrate Ridge of the Cascadia margin shows that the carbonate formation may be related to the dynamic formation and dissociation of gas hydrates due to its fragile stability [41]. However, the relationship of gas hydrates and carbonates is in substance related to the fluid source, and it was proposed that prolific methane seepage will lead to increased AOM efficiency [63]. For example, researchers found that $10 \mathrm{~m}$ high ridges of methane-derived authigenic carbonate rocks developed within a large pockmark in mid-Norway [70] (Figures 5(a) and 5(b)). The amount of authigenic carbonates is comprehensively determined by the methane supply and the rate of AOM and the duration time [71]. Not all pockmarks are accompanied by authigenic carbonates. For example, in the northern Carnarvon basin offshore 


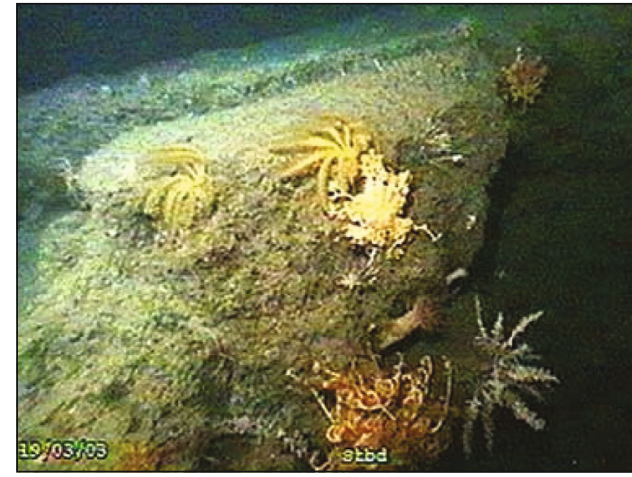

(a)

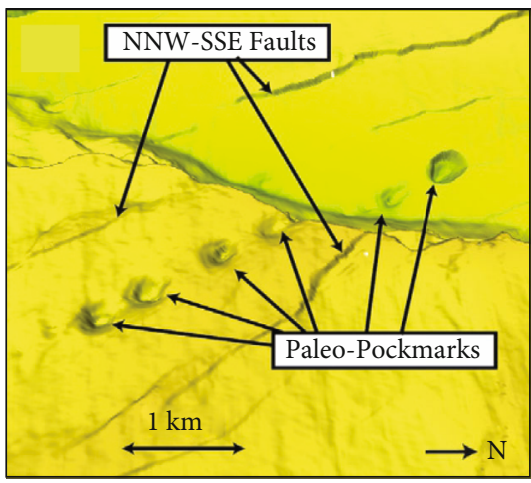

(c)

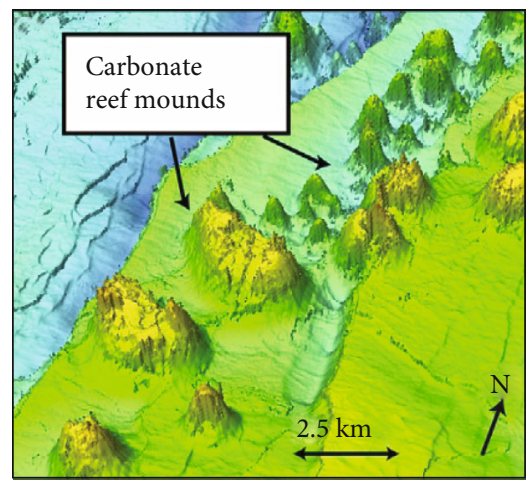

(e)

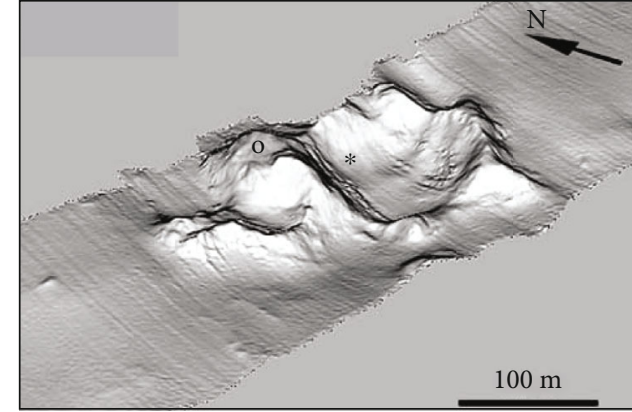

(b)

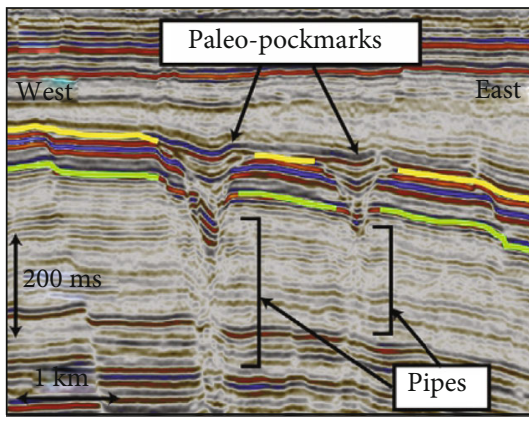

(d)

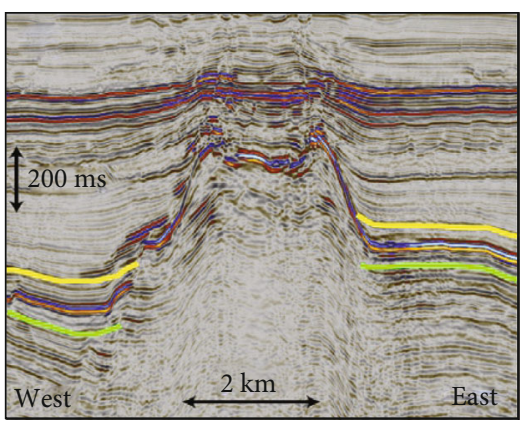

(f)

FIGURE 5: (a) Photo of carbonates at the seabed observed by ROV located within a large pockmark as shown in (b) (modified from Hovland et al. [70]). (b) A large pockmark with carbonates developed within it in mid-Norway (modified from Hovland et al. [70]). (c) Plan view map showing the linear distribution of paleopockmarks in Exmouth Plateau, Northern Carnarvon Basin (modified from Velayatham et al. [61]). (d) A seismic profile showing the paleopockmarks in Exmouth Plateau, Northern Carnarvon Basin (modified from Velayatham et al. [61]). (e) Plan view map showing the carbonates in Exmouth Plateau, Northern Carnarvon Basin (modified from Velayatham et al. [61]). (f) Seismic profile showing the positive relief of carbonates in Exmouth Plateau, Northern Carnarvon Basin (modified from Velayatham et al. [61]).

northwest Australia, the pockmarks in the east are not accompanied by carbonate rocks, while the pockmarks in the west are accompanied by carbonate rocks [61] (Figures 5(c)-5(f)).

In Table 1, we supplement the pockmark compilation of Panieri et al. [53] by referring to more published global case studies, displaying information on water depth, pockmark formation mechanism including relevant fluid pathways and gas source, nearby carbonates, and relationship with the gas hydrate system. Compilation shows that $62.2 \%$ pockmarks are accompanied by authigenic carbonates, and the preponderance of pockmarks coexists with the gas hydrate systems or around the LLGHSZ. According to previous studies, the pockmark density and total number of pockmarks are the largest around the LLGHSZ which has been observed in Arctic continental shelves [72, 73], US Atlantic margin [18-20], and the West Spitsbergen continental margin [21]. 
TABLE 1: Compilation of pockmark and carbonate locations around the world showing formation mechanisms and relationship with the gas hydrate systems (modified from Panieri et al. [53]).

\begin{tabular}{|c|c|c|c|c|c|}
\hline Pockmark location & $\begin{array}{l}\text { Water } \\
\text { depth }\end{array}$ & Formation mechanisms & Carbonates & $\begin{array}{l}\text { Relationship with } \\
\text { the gas hydrate } \\
\text { system }\end{array}$ & References \\
\hline $\begin{array}{l}\text { Arctic Ocean (Vestnesa } \\
\text { Ridge) }\end{array}$ & $1200 \mathrm{~m}$ & $\begin{array}{l}\text { Fluid pathways: faults, chimneys, and fractures } \\
\text { Gas source: deep reservoir and shallow free gas }\end{array}$ & $\begin{array}{l}\text { Carbonates of } \\
\text { various shapes }\end{array}$ & $\begin{array}{l}\text { Deeper than the } \\
\text { LLGHSZ }\end{array}$ & $\begin{array}{l}{[60,74} \\
75]\end{array}$ \\
\hline $\begin{array}{l}\text { Arctic Ocean (Spitsbergen } \\
\text { fjords) }\end{array}$ & $\begin{array}{c}110- \\
130 \mathrm{~m}\end{array}$ & $\begin{array}{l}\text { Fluid pathways: tectonic controls (faults) } \\
\text { Gas source: thermogenic gas from the organic- } \\
\text { rich shales }\end{array}$ & No report & $\begin{array}{l}\text { Shallower than } \\
\text { the GHSZ }\end{array}$ & {$[76]$} \\
\hline Barents Sea & $\begin{array}{c}200- \\
1270 \mathrm{~m}\end{array}$ & $\begin{array}{c}\text { Fluid pathways: gas pressure and faults } \\
\text { Gas source: thermogenic gas, hydrate } \\
\text { dissociation }\end{array}$ & $\begin{array}{l}\text { Authigenic } \\
\text { carbonates }\end{array}$ & $\begin{array}{l}\text { Gas hydrates at } \\
\text { different depths }\end{array}$ & {$[77-80]$} \\
\hline Bering Sea (Alaska) & $\begin{array}{c}< \\
200 \mathrm{~m}\end{array}$ & $\begin{array}{l}\text { Fluid pathways: faults and fractures } \\
\text { Gas source: biogenic and thermogenic gas }\end{array}$ & No report & $\begin{array}{l}\text { Shallower than } \\
\text { the GHSZ }\end{array}$ & {$[81]$} \\
\hline $\begin{array}{l}\text { Norwegian Sea (Troll } \\
\text { region) }\end{array}$ & $300 \mathrm{~m}$ & $\begin{array}{c}\text { Fluid pathways: gas hydrate dissociation } \\
\text { Gas source: gas hydrate }\end{array}$ & $\begin{array}{l}\text { Authigenic } \\
\text { carbonates }\end{array}$ & $\begin{array}{l}\text { Shallower than } \\
\text { the GHSZ }\end{array}$ & {$[82]$} \\
\hline $\begin{array}{l}\text { Norwegian Sea } \\
\text { (continental shelf and } \\
\text { slope) }\end{array}$ & $\begin{array}{c}300- \\
800 \mathrm{~m}\end{array}$ & $\begin{array}{c}\text { Fluid pathways: overpressure, chimneys, and } \\
\text { fractures } \\
\text { Gas source: gas reservoir and free gas }\end{array}$ & Carbonate ridges & $\begin{array}{l}\text { Shallower than } \\
\text { the GHSZ } \\
\text { Around LLGHSZ }\end{array}$ & {$[83]$} \\
\hline $\begin{array}{l}\text { Norwegian Sea (Nyegga } \\
\text { region) }\end{array}$ & $\begin{array}{c}500- \\
1500 \mathrm{~m}\end{array}$ & $\begin{array}{l}\text { Fluid pathways: chimneys and faults } \\
\text { Gas source: gas reservoir and free gas }\end{array}$ & $\begin{array}{l}\text { Carbonates of } \\
\text { various shapes }\end{array}$ & $\begin{array}{l}\text { Around LLGHSZ } \\
\text { Deeper than the } \\
\text { LLGHSZ }\end{array}$ & {$[84]$} \\
\hline NE Atlantic margin & $\begin{array}{c}400- \\
1500 \mathrm{~m}\end{array}$ & $\begin{array}{l}\text { Fluid pathways: gas hydrate dissociation } \\
\text { Gas source: gas hydrate }\end{array}$ & No report & $\begin{array}{l}\text { Around LLGHSZ } \\
\text { Deeper than the } \\
\text { LLGHSZ }\end{array}$ & {$[85]$} \\
\hline $\begin{array}{l}\text { Strait between Norway and } \\
\text { Denmark (Skagerrak) }\end{array}$ & $\begin{array}{c}100- \\
200 \mathrm{~m}\end{array}$ & $\begin{array}{l}\text { Fluid pathways: clay diapir and faults } \\
\text { Gas source: biogenic and thermogenic gas }\end{array}$ & $\begin{array}{l}\text { Carbonate } \\
\text { cemented }\end{array}$ & $\begin{array}{l}\text { Shallower than } \\
\text { the GHSZ }\end{array}$ & {$[86]$} \\
\hline Canada (Pacific margin) & $\begin{array}{c}130- \\
250 \mathrm{~m}\end{array}$ & $\begin{array}{c}\text { Fluid pathways: fault formation inside iceberg } \\
\text { ploughmarks } \\
\text { Gas source: hydrocarbon gas }\end{array}$ & $\begin{array}{l}\text { Authigenic } \\
\text { carbonates, } \\
\text { carbonate ridges }\end{array}$ & $\begin{array}{l}\text { Shallower than } \\
\text { the GHSZ }\end{array}$ & {$[87,88]$} \\
\hline $\begin{array}{l}\text { North Sea, UK sector } \\
\text { (Witch Ground Basin) }\end{array}$ & $\begin{array}{c}100- \\
150 \mathrm{~m}\end{array}$ & $\begin{array}{l}\text { Fluid pathways: degradation of subseabed } \\
\text { permafrost ice } \\
\text { Gas source: biogenic and thermogenic gas }\end{array}$ & No report & $\begin{array}{l}\text { Shallower than } \\
\text { the GHSZ }\end{array}$ & [89] \\
\hline $\begin{array}{l}\text { Atlantic Ocean (Belfast } \\
\text { Bay, Maine) }\end{array}$ & $30 \mathrm{~m}$ & $\begin{array}{l}\text { Fluid pathways: structure } \\
\text { Gas source: biogenic natural gas and pore water }\end{array}$ & No report & $\begin{array}{l}\text { Shallower than } \\
\text { the GHSZ }\end{array}$ & {$[90]$} \\
\hline $\begin{array}{l}\text { Pacific Ocean (Big Sur, } \\
\text { California) }\end{array}$ & $\begin{array}{c}900- \\
1200 \mathrm{~m}\end{array}$ & $\begin{array}{l}\text { Fluid pathways: no report } \\
\text { Gas source: free gas }\end{array}$ & $\begin{array}{l}\text { Biogenic } \\
\text { carbonate } \\
\text { fragments }\end{array}$ & $\begin{array}{l}\text { Deeper than the } \\
\text { LLGHSZ }\end{array}$ & [91] \\
\hline Gulf of Mexico & $\begin{array}{c}100- \\
500 \mathrm{~m}\end{array}$ & $\begin{array}{l}\text { Fluid pathways: fault } \\
\text { Gas source: thermogenic gas }\end{array}$ & $\begin{array}{l}\text { Carbonate } \\
\text { buildups }\end{array}$ & $\begin{array}{l}\text { Shallower than } \\
\text { the GHSZ } \\
\text { Around LLGHSZ }\end{array}$ & {$[92]$} \\
\hline $\begin{array}{l}\text { Atlantic Ocean (Santos } \\
\text { Basin) }\end{array}$ & $\begin{array}{c}400- \\
900 \mathrm{~m}\end{array}$ & $\begin{array}{c}\text { Fluid pathways: faults and salt diapirism } \\
\text { Gas source: salt diapirs and hydrocarbon } \\
\text { province }\end{array}$ & No report & $\begin{array}{l}\text { Around LLGHSZ } \\
\text { Deeper than the } \\
\text { LLGHSZ }\end{array}$ & {$[52,93]$} \\
\hline SW Africa (Niger Delta) & $\begin{array}{c}1100- \\
1250 \mathrm{~m}\end{array}$ & $\begin{array}{c}\text { Fluid pathways: faults, overpressure, hydrate } \\
\text { dissociation } \\
\text { Gas source: hydrate dissociation }\end{array}$ & $\begin{array}{l}\text { Carbonate rock } \\
\text { crust }\end{array}$ & $\begin{array}{l}\text { Deeper than the } \\
\text { LLGHSZ }\end{array}$ & {$[94]$} \\
\hline $\begin{array}{l}\text { SW Africa (Congo deep- } \\
\text { sea channel) }\end{array}$ & $3160 \mathrm{~m}$ & $\begin{array}{l}\text { Fluid pathways: faults, chimneys, and fractures } \\
\text { Gas source: no report }\end{array}$ & Carbonate crusts & $\begin{array}{l}\text { Deeper than the } \\
\text { LLGHSZ }\end{array}$ & {$[95]$} \\
\hline $\begin{array}{l}\text { SW Africa (Northern } \\
\text { Congo Fan) }\end{array}$ & $3100 \mathrm{~m}$ & $\begin{array}{c}\text { Fluid pathways: fault zones, salt diapirs, } \\
\text { polygonal faults } \\
\text { Gas source: biogenic gas }\end{array}$ & $\begin{array}{l}\text { Carbonates of } \\
\text { various shapes }\end{array}$ & $\begin{array}{l}\text { Deeper than the } \\
\text { LLGHSZ }\end{array}$ & {$[96,97]$} \\
\hline $\begin{array}{l}\text { South China Sea }(\mathrm{N} \\
\text { Zhongjiannan Basin) }\end{array}$ & $\begin{array}{c}600- \\
1400 \mathrm{~m}\end{array}$ & $\begin{array}{l}\text { Fluid pathways: listric faults, chimneys } \\
\text { Gas source: hydrocarbon-rich fluids }\end{array}$ & $\begin{array}{l}\text { Carbonate } \\
\text { cements }\end{array}$ & $\begin{array}{l}\text { Deeper than the } \\
\text { LLGHSZ }\end{array}$ & {$[98]$} \\
\hline $\begin{array}{l}\text { South China Sea (Xisha } \\
\text { Uplift) }\end{array}$ & $700 \mathrm{~m}$ & $\begin{array}{l}\text { Fluid pathways: faults, chimney } \\
\text { Gas source: deep reservoir }\end{array}$ & No report & $\begin{array}{l}\text { Deeper than the } \\
\text { LLGHSZ }\end{array}$ & [99] \\
\hline
\end{tabular}


TABLE 1: Continued.

\begin{tabular}{|c|c|c|c|c|c|}
\hline Pockmark location & $\begin{array}{l}\text { Water } \\
\text { depth }\end{array}$ & Formation mechanisms & Carbonates & $\begin{array}{l}\text { Relationship with } \\
\text { the gas hydrate } \\
\text { system }\end{array}$ & References \\
\hline E Atlantic (Gulf of Cadiz) & $\begin{array}{c}300- \\
400 \mathrm{~m}\end{array}$ & $\begin{array}{l}\text { Fluid pathways: faults, chimney, and diapir } \\
\text { Gas source: biogenic and thermogenic gas }\end{array}$ & $\begin{array}{l}\text { Authigenic } \\
\text { carbonates }\end{array}$ & $\begin{array}{l}\text { Shallower than } \\
\text { the GHSZ }\end{array}$ & {$[100]$} \\
\hline $\begin{array}{l}\text { Central Mediterranean } \\
\text { (Zannone Giant } \\
\text { Pockmark) }\end{array}$ & $\begin{array}{c}110- \\
130 \mathrm{~m}\end{array}$ & $\begin{array}{l}\text { Fluid pathways: faults } \\
\text { Fluid pathways: no report }\end{array}$ & $\begin{array}{l}\text { Authigenic } \\
\text { carbonates }\end{array}$ & $\begin{array}{l}\text { Shallower than } \\
\text { the GHSZ }\end{array}$ & {$[101]$} \\
\hline $\begin{array}{l}\text { W Mediterranean Sea } \\
\text { (TASYO field, Gulf of } \\
\text { Cadiz) }\end{array}$ & $\begin{array}{c}500- \\
2000 \mathrm{~m}\end{array}$ & $\begin{array}{l}\text { Fluid pathways: episodic dissociation of gas- } \\
\text { hydrate-rich sediments, tectonic and climate/ } \\
\text { oceanographic factors } \\
\text { Gas source: petrogenic gas }\end{array}$ & Carbonate crusts & $\begin{array}{l}\text { Around LLGHSZ } \\
\text { Deeper than the } \\
\text { LLGHSZ }\end{array}$ & {$[102]$} \\
\hline $\begin{array}{l}\text { SW Mediterranean Sea } \\
\text { (Gulf of Cadiz) }\end{array}$ & $\begin{array}{c}200- \\
400 \mathrm{~m}\end{array}$ & $\begin{array}{l}\text { Fluid pathways: faults, sediment lithology } \\
\text { Gas source: biogenic and thermogenic gas }\end{array}$ & No report & $\begin{array}{l}\text { Shallower than } \\
\text { the GHSZ }\end{array}$ & [103] \\
\hline $\begin{array}{l}\text { E Mediterranean Sea } \\
\text { (Patras Gulf, Greece) }\end{array}$ & $\begin{array}{c}20- \\
80 \mathrm{~m}\end{array}$ & $\begin{array}{l}\text { Fluid pathways: faults, earthquake } \\
\text { Gas source: no report }\end{array}$ & No report & $\begin{array}{l}\text { Shallower than } \\
\text { the GHSZ }\end{array}$ & {$[104]$} \\
\hline $\begin{array}{l}\text { E Mediterranean Sea } \\
\text { (Patras and Corinth gulfs, } \\
\text { Greece) }\end{array}$ & $\begin{array}{c}20- \\
40 \mathrm{~m}\end{array}$ & $\begin{array}{c}\text { Fluid pathways: gas venting and groundwater } \\
\text { seepage } \\
\text { Gas source: underground water }\end{array}$ & No report & $\begin{array}{l}\text { Shallower than } \\
\text { the GHSZ }\end{array}$ & [105] \\
\hline $\begin{array}{l}\text { E Mediterranean Sea } \\
\text { (Iskenderun Bay) }\end{array}$ & $70 \mathrm{~m}$ & $\begin{array}{l}\text { Fluid pathways: faults } \\
\text { Gas source: no report }\end{array}$ & Buried carbonate & $\begin{array}{l}\text { Shallower than } \\
\text { the GHSZ }\end{array}$ & {$[106]$} \\
\hline $\begin{array}{l}\text { NW Black Sea (Dnepr } \\
\text { paleodelta) }\end{array}$ & $90 \mathrm{~m}$ & $\begin{array}{l}\text { Fluid pathways: no report } \\
\text { Gas source: free gas }\end{array}$ & $\begin{array}{l}\text { Carbonate } \\
\text { cemented }\end{array}$ & $\begin{array}{l}\text { Shallower than } \\
\text { the GHSZ }\end{array}$ & {$[107]$} \\
\hline E Black Sea & $\begin{array}{c}100- \\
2000 \mathrm{~m}\end{array}$ & $\begin{array}{l}\text { Fluid pathways: local subsidence, faults } \\
\text { Gas source: shallow gas }\end{array}$ & No report & $\begin{array}{l}\text { Hydrates at } \\
\text { different depths }\end{array}$ & [108] \\
\hline Western Indian margin & $\begin{array}{c}20- \\
260 \mathrm{~m}\end{array}$ & $\begin{array}{c}\text { Fluid pathways: deep-seated faults, collapse } \\
\text { structures, dissociation of gas hydrates } \\
\text { Gas source: thermogenic gas }\end{array}$ & No report & $\begin{array}{l}\text { Shallower than } \\
\text { the GHSZ }\end{array}$ & [109] \\
\hline $\begin{array}{l}\text { NW Shelf of Australia } \\
\text { (Yampi Shelf and Timor } \\
\text { Sea region) }\end{array}$ & $\begin{array}{c}50- \\
100 \mathrm{~m}\end{array}$ & $\begin{array}{l}\text { Fluid pathways: preexisting fractures and dykes } \\
\text { Gas source: no report }\end{array}$ & $\begin{array}{c}\text { Crusts of } \\
\text { cemented } \\
\text { bioclastic } \\
\text { material }\end{array}$ & $\begin{array}{l}\text { Shallower than } \\
\text { the GHSZ }\end{array}$ & {$[110]$} \\
\hline $\begin{array}{l}\text { Northwest Australia } \\
\text { (Exmouth Plateau) }\end{array}$ & $\begin{array}{c}20- \\
95 \mathrm{~m}\end{array}$ & $\begin{array}{l}\text { Fluid pathways: faults, overpressure } \\
\text { Gas source: no report }\end{array}$ & $\begin{array}{l}\text { Carbonate reef } \\
\text { mounds }\end{array}$ & $\begin{array}{l}\text { Shallower than } \\
\text { the GHSZ }\end{array}$ & {$[61]$} \\
\hline $\begin{array}{l}\text { South China Sea } \\
\text { (Yinggehai Basin) }\end{array}$ & $\begin{array}{c}10- \\
50 \mathrm{~m}\end{array}$ & $\begin{array}{l}\text { Fluid pathways: faults and diapir, coarse-grained } \\
\text { sediments, unconformity, fractures } \\
\text { Gas source: hydrocarbons in the sediments }\end{array}$ & No report & $\begin{array}{l}\text { Shallower than } \\
\text { the GHSZ }\end{array}$ & {$[111]$} \\
\hline $\begin{array}{l}\text { South China Sea (SW of } \\
\text { Qiongdongnan Basin) }\end{array}$ & $\begin{array}{c}600- \\
1600 \mathrm{~m}\end{array}$ & $\begin{array}{l}\text { Fluid pathways: gas chimney and faults } \\
\text { Gas source: free gas, thermogenic gas }\end{array}$ & $\begin{array}{l}\text { Authigenic } \\
\text { carbonates }\end{array}$ & $\begin{array}{l}\text { Deeper than the } \\
\text { LLGHSZ }\end{array}$ & {$[112]$} \\
\hline $\begin{array}{l}\text { Western margin of the } \\
\text { South China Sea } \\
\text { (Zhongjiannan Basin) }\end{array}$ & $\begin{array}{c}900- \\
1100 \mathrm{~m}\end{array}$ & $\begin{array}{l}\text { Fluid pathways: extensional fault, the thermal } \\
\text { subsidence fault, and uplifting fault } \\
\text { Gas source: deep reservoir, free gas }\end{array}$ & $\begin{array}{l}\text { Carbonate reef } \\
\text { mounds }\end{array}$ & $\begin{array}{l}\text { Deeper than the } \\
\text { LLGHSZ }\end{array}$ & [113] \\
\hline $\begin{array}{l}\text { South China Sea (northern } \\
\text { Qiongdongnan Basin) }\end{array}$ & $\begin{array}{l}1600- \\
1800 \mathrm{~m}\end{array}$ & $\begin{array}{l}\text { The fractures in the seepage pathways provided } \\
\text { direct evidence of vertical fluid flow }\end{array}$ & $\begin{array}{l}\text { Authigenic } \\
\text { carbonates }\end{array}$ & $\begin{array}{l}\text { Deeper than the } \\
\text { LLGHSZ }\end{array}$ & {$[114]$} \\
\hline $\begin{array}{l}\text { Northeast Atlantic } \\
\text { (northern Rockall Trough) }\end{array}$ & $\begin{array}{c}900- \\
1000 \mathrm{~m}\end{array}$ & $\begin{array}{l}\text { Fluid pathways: faults, overpressure } \\
\text { Gas source: pore water }\end{array}$ & No report & $\begin{array}{l}\text { Deeper than the } \\
\text { LLGHSZ }\end{array}$ & [115] \\
\hline
\end{tabular}

3.2. Deep-Water Corals. Deep-water corals, also known as cold-water corals, were first described in the $18^{\text {th }}$ century [116], but until the late $20^{\text {th }}$ century were the deep-water corals generally delineated with the development of submersibles [117]. They often live without light in relatively nutrient-rich seawater and are independent of symbiotic algae. Deep-water corals commonly occur at water depth ranging from 39 to over $2000 \mathrm{~m}$, which cover the latitude ranging from $70^{\circ} \mathrm{N}$ to $60^{\circ} \mathrm{S}$, with temperature ranging from
0 to $13.6^{\circ} \mathrm{C}$ [118]. Till now, deep-water corals have been discovered at many locations all over the world (Figure 1(b); Table 2), such as off mid-Norway [118], North Sea [119], South China Sea [120], Mediterranean Sea [121], Atlantic Ocean [122, 123], and offshore Mauritania [124]. Most studies of deep-water corals focused on their biology and role in global climate change, while limited has been done about their formation mechanisms. Hovland and Thomsen [118] proposed that the deep-water corals in off mid-Norway are 
TABLE 2: Compilation of deep-water coral locations around the world showing information on water depth, size, coral types, relationship with the gas hydrate system, and related fluid seepage.

\begin{tabular}{|c|c|c|c|c|c|c|}
\hline $\begin{array}{l}\text { Deep-water coral } \\
\text { location }\end{array}$ & $\begin{array}{l}\text { Water } \\
\text { depth }\end{array}$ & Size & Types & $\begin{array}{c}\text { Relationship } \\
\text { with gas } \\
\text { hydrate system }\end{array}$ & $\begin{array}{l}\text { Related fluid } \\
\text { seepage }\end{array}$ & References \\
\hline $\begin{array}{l}\text { Norway (northern, } \\
\text { mid, southwest) }\end{array}$ & $\begin{array}{c}39- \\
400 \mathrm{~m}\end{array}$ & $\begin{array}{c}\text { Height: } 45 \mathrm{~m} \text {, width: } \\
>100 \mathrm{~m}\end{array}$ & L. pertusa & $\begin{array}{l}\text { Shallower than } \\
\text { the GHSZ }\end{array}$ & Hydrocarbon seeps & {$[140]$} \\
\hline $\begin{array}{l}\text { New Zealand } \\
\text { (Hikurangi Margin) }\end{array}$ & $\begin{array}{c}756- \\
1120 \mathrm{~m}\end{array}$ & Coral ridge & $\begin{array}{l}\text { Desmophyllum, } \\
\text { Caryophyllia }\end{array}$ & $\begin{array}{l}\text { Deeper than } \\
\text { the LLGHSZ }\end{array}$ & $\begin{array}{c}\text { Gas seeps } \\
\text { Authigenic } \\
\text { carbonates } \\
\text { Chemosynthetic } \\
\text { fauna }\end{array}$ & {$[141,142]$} \\
\hline $\begin{array}{l}\text { Gulf of Cádiz } \\
\text { (Guadalquivir } \\
\text { Diapiric Ridge) }\end{array}$ & $\begin{array}{c}800- \\
1100 \mathrm{~m}\end{array}$ & No report & $\begin{array}{l}\text { L. pertusa } \\
\text { M. oculata }\end{array}$ & $\begin{array}{l}\text { Deeper than } \\
\text { the LLGHSZ }\end{array}$ & $\begin{array}{l}\text { "Invasions" of the } \\
\text { warmer } \\
\text { Mediterranean } \\
\text { fauna }\end{array}$ & {$[143]$} \\
\hline $\begin{array}{l}\text { Gulf of Mexico (De } \\
\text { Soto Slope) }\end{array}$ & $\begin{array}{c}434- \\
530 \mathrm{~m}\end{array}$ & $\begin{array}{l}\text { Height and width: } 1.5- \\
2 \mathrm{~m} \text {, length: } 3-4 \mathrm{~m} \\
\text { diameter: }>1.5 \mathrm{~m}\end{array}$ & L. pertusa & $\begin{array}{l}\text { Around } \\
\text { LLGHSZ }\end{array}$ & $\begin{array}{l}\text { Active hydrocarbon } \\
\text { seepage } \\
\text { Carbonate knolls } \\
\text { and ridges }\end{array}$ & {$[144]$} \\
\hline Norwegian coast & $\begin{array}{c}39- \\
550 \mathrm{~m}\end{array}$ & $\begin{array}{c}\text { Height: } 2-33 \mathrm{~m}, \\
\text { diameter: } 50-500 \mathrm{~m}\end{array}$ & L. pertusa & $\begin{array}{c}\text { Shallower than } \\
\text { the GHSZ } \\
\text { Around } \\
\text { LLGHSZ }\end{array}$ & Iceberg plough & {$[145]$} \\
\hline $\begin{array}{l}\text { Canada (Jordan } \\
\text { basin) }\end{array}$ & $\begin{array}{c}154- \\
1400 \mathrm{~m}\end{array}$ & No report & L. pertusa & $\begin{array}{l}\text { Gas hydrates at } \\
\text { different depths }\end{array}$ & $\begin{array}{l}\text { Hydrodynamic } \\
\text { regime }\end{array}$ & {$[146]$} \\
\hline $\begin{array}{l}\text { NE Atlantic } \\
\text { (Porcupine Seabight) }\end{array}$ & $\begin{array}{c}798- \\
942 \mathrm{~m}\end{array}$ & $\begin{array}{l}\text { Height: } 100-140 \mathrm{~m} \text {, } \\
\text { width: } 650 \mathrm{~m} \text {, length: } \\
1.3 \mathrm{~km}\end{array}$ & $\begin{array}{l}\text { L. pertusa } \\
\text { M. oculata }\end{array}$ & $\begin{array}{l}\text { Deeper than } \\
\text { the LLGHSZ }\end{array}$ & Carbonates & [147] \\
\hline $\begin{array}{l}\text { Irish margin (Rockall } \\
\text { Bank) }\end{array}$ & $\begin{array}{c}100- \\
1000 \mathrm{~m}\end{array}$ & No report & No report & $\begin{array}{l}\text { Gas hydrates at } \\
\text { different depths }\end{array}$ & Hiatuses & [123] \\
\hline $\begin{array}{l}\text { SE U.S. (Blake } \\
\text { Plateau) }\end{array}$ & $\begin{array}{c}640- \\
869 \mathrm{~m}\end{array}$ & Height: $146 \mathrm{~m}$ & L. pertusa & $\begin{array}{l}\text { Deeper than } \\
\text { the LLGHSZ }\end{array}$ & No report & {$[148]$} \\
\hline $\begin{array}{l}\text { SE U.S. (Straits of } \\
\text { Florida) }\end{array}$ & $\begin{array}{c}490- \\
550 \mathrm{~m}\end{array}$ & Height: $15-23 \mathrm{~m}$ & Octocorallia & $\begin{array}{l}\text { Around } \\
\text { LLGHSZ }\end{array}$ & $\begin{array}{l}\text { Strong bottom } \\
\text { currents }\end{array}$ & {$[148]$} \\
\hline $\begin{array}{l}\text { SE U.S. (eastern Gulf } \\
\text { of Mexico) }\end{array}$ & $780 \mathrm{~m}$ & Height: $140 \mathrm{~m}$ & No report & $\begin{array}{l}\text { Deeper than } \\
\text { the LLGHSZ }\end{array}$ & No report & [148] \\
\hline $\begin{array}{l}\text { Gulf of Mexico } \\
\text { (upper continental } \\
\text { slope) }\end{array}$ & $\begin{array}{c}315- \\
535 \mathrm{~m}\end{array}$ & No report & L. pertusa & $\begin{array}{c}\text { Shallower than } \\
\text { the GHSZ } \\
\text { Around } \\
\text { LLGHSZ }\end{array}$ & Gas seeps & [149] \\
\hline $\begin{array}{l}\text { Uruguayan (outer } \\
\text { shelf and slope) }\end{array}$ & $\begin{array}{c}167- \\
326 \mathrm{~m}\end{array}$ & Height: $\sim 35 \mathrm{~m}$ & No report & $\begin{array}{l}\text { Shallower than } \\
\text { the GHSZ }\end{array}$ & Gas seeps & {$[122]$} \\
\hline SW Australia & $\begin{array}{c}982- \\
2395 \mathrm{~m}\end{array}$ & No report & $\begin{array}{c}\text { Corallium, Paragorgia, D. } \\
\text { dianthus }\end{array}$ & $\begin{array}{l}\text { Deeper than } \\
\text { the LLGHSZ }\end{array}$ & No report & {$[150]$} \\
\hline $\begin{array}{l}\text { New Zealand (region } \\
\text { seamounts) }\end{array}$ & $\begin{array}{c}200- \\
2850 \mathrm{~m}\end{array}$ & No report & $\begin{array}{c}\text { Oculina, M. oculata, O. } \\
\text { virgosa }\end{array}$ & $\begin{array}{l}\text { Gas hydrates at } \\
\text { different depths }\end{array}$ & $\begin{array}{l}\text { Strong bottom } \\
\text { currents }\end{array}$ & {$[151]$} \\
\hline $\begin{array}{l}\text { Bay of Biscay } \\
\text { (Whittard Canyon) }\end{array}$ & $\begin{array}{c}880- \\
3300 \mathrm{~m}\end{array}$ & No report & $\begin{array}{l}\text { Anthomastus spp. } \\
\text { L. pertusa } \\
\text { Octocorals } \\
\text { Primnoa spp. }\end{array}$ & $\begin{array}{l}\text { Deeper than } \\
\text { the LLGHSZ }\end{array}$ & $\begin{array}{l}\text { Strong bottom } \\
\text { currents }\end{array}$ & {$[152,153]$} \\
\hline Mid-Atlantic Ridge & $\begin{array}{c}800- \\
2400 \mathrm{~m}\end{array}$ & No report & Octocorals & $\begin{array}{l}\text { Deeper than } \\
\text { the LLGHSZ }\end{array}$ & $\begin{array}{l}\text { Strong bottom } \\
\text { currents }\end{array}$ & {$[154]$} \\
\hline $\begin{array}{l}\text { Central } \\
\text { Mediterranean Sea }\end{array}$ & $\begin{array}{c}100- \\
1600 \mathrm{~m}\end{array}$ & Height: <1 m & White coral & $\begin{array}{l}\text { Gas hydrates at } \\
\text { different depths }\end{array}$ & No report & {$[155]$} \\
\hline $\begin{array}{l}\text { Offshore Mauritania, } \\
\text { Northwest Africa }\end{array}$ & $\begin{array}{l}450- \\
550 \mathrm{~m}\end{array}$ & Width: $500 \mathrm{~m}$ & & $\begin{array}{l}\text { Around } \\
\text { LLGHSZ }\end{array}$ & Gas seeps & {$[124]$} \\
\hline
\end{tabular}


TABLE 2: Continued.

\begin{tabular}{|c|c|c|c|c|c|c|}
\hline $\begin{array}{l}\text { Deep-water coral } \\
\text { location }\end{array}$ & $\begin{array}{l}\text { Water } \\
\text { depth }\end{array}$ & Size & Types & $\begin{array}{c}\text { Relationship } \\
\text { with gas } \\
\text { hydrate system } \\
\end{array}$ & $\begin{array}{l}\text { Related fluid } \\
\text { seepage }\end{array}$ & References \\
\hline & & & $\begin{array}{l}\text { L. pertusa, M. oculata, } \\
\text { Solenosmilia variabilis, } \\
\text { Desmophyllum }\end{array}$ & & & \\
\hline $\begin{array}{l}\text { Off southwest Ireland } \\
\text { (Porcupine Basin) }\end{array}$ & $\begin{array}{c}200- \\
2000 \mathrm{~m}\end{array}$ & $\begin{array}{l}\text { Height: } 200 \mathrm{~m} \text {, width: } \\
1 \mathrm{~km} \text {, length: } 5 \mathrm{~km}\end{array}$ & Coral mound & $\begin{array}{l}\text { Gas hydrates at } \\
\text { different depths }\end{array}$ & Bottom currents & {$[156]$} \\
\hline $\begin{array}{l}\text { Southern Rockall } \\
\text { Trough }\end{array}$ & $\begin{array}{c}500- \\
1200 \mathrm{~m}\end{array}$ & $\begin{aligned} \text { Height: } & 350 \mathrm{~m} \text {, width: } \\
& 2 \mathrm{~km}\end{aligned}$ & Coral mound & $\begin{array}{l}\text { Around } \\
\text { LLGHSZ } \\
\text { Deeper than } \\
\text { the LLGHSZ }\end{array}$ & Bottom currents & {$[157]$} \\
\hline $\begin{array}{l}\text { Agdenes morainic } \\
\text { ridge }\end{array}$ & $\begin{array}{c}150- \\
500 \mathrm{~m}\end{array}$ & Width: $100 \mathrm{~m}$ & L. pertusa & $\begin{array}{c}\text { Shallower than } \\
\text { the GHSZ } \\
\text { Around } \\
\text { LLGHSZ }\end{array}$ & Gas chimneys & {$[158]$} \\
\hline Sula Ridge & $\begin{array}{c}233- \\
330 \mathrm{~m}\end{array}$ & $\begin{array}{l}\text { Height: } 45 \text { km, length: } \\
13 \mathrm{~km}\end{array}$ & L. pertusa & $\begin{array}{l}\text { Shallower than } \\
\text { the GHSZ }\end{array}$ & Gas chimneys & [159] \\
\hline $\begin{array}{l}\text { Off mid-Norway (east } \\
\text { Draugen field) }\end{array}$ & $280 \mathrm{~m}$ & No report & L. pertusa & $\begin{array}{l}\text { Shallower than } \\
\text { the GHSZ }\end{array}$ & Gas seeps & {$[158]$} \\
\hline $\begin{array}{l}\text { Norwegian } \\
\text { Concession Block } \\
\text { (island Træna) }\end{array}$ & $\begin{array}{c}300- \\
330 \mathrm{~m}\end{array}$ & $\begin{array}{l}\text { The largest: height: } 15 \mathrm{~m} \text {, } \\
\text { length: } 700 \mathrm{~m} \text {, width: } \\
150 \mathrm{~m}\end{array}$ & L. pertusa & $\begin{array}{l}\text { Shallower than } \\
\text { the GHSZ }\end{array}$ & Gas chimneys & {$[158]$} \\
\hline $\begin{array}{l}\text { Off mid-Norway } \\
\text { (Kristin hydrocarbon } \\
\text { field) }\end{array}$ & $\begin{array}{c}310- \\
385 \mathrm{~m}\end{array}$ & $\begin{array}{l}\text { The largest: height: } \\
3.5 \mathrm{~m} \text {, length: } 90 \mathrm{~m}\end{array}$ & No report & $\begin{array}{l}\text { Shallower than } \\
\text { the GHSZ }\end{array}$ & No report & {$[70]$} \\
\hline $\begin{array}{l}\text { Between Norway and } \\
\text { UK (Langeled } \\
\text { pipeline) }\end{array}$ & $280 \mathrm{~m}$ & No report & L. pertusa & $\begin{array}{l}\text { Shallower than } \\
\text { the GHSZ }\end{array}$ & No report & {$[158]$} \\
\hline Vestera ${ }^{\circ}$ lsgrunnen & $\begin{array}{c}200- \\
270 \mathrm{~m}\end{array}$ & $\begin{array}{l}\text { Height: } 20 \text { m, width: } 150- \\
200 \mathrm{~m}\end{array}$ & No report & $\begin{array}{l}\text { Shallower than } \\
\text { the GHSZ }\end{array}$ & Bottom currents & {$[158]$} \\
\hline West Skogsøy & $200 \mathrm{~m}$ & No report & Floholman reefs & $\begin{array}{l}\text { Shallower than } \\
\text { the GHSZ }\end{array}$ & $\begin{array}{c}\text { Gas seeps, bacterial } \\
\text { mats }\end{array}$ & {$[158]$} \\
\hline Storegga slope & $\begin{array}{c}300- \\
400 \mathrm{~m}\end{array}$ & $\begin{array}{l}\text { Width: } 3 \mathrm{~km} \text {, length: } \\
35 \mathrm{~km}\end{array}$ & L. pertusa & $\begin{array}{l}\text { Shallower than } \\
\text { the GHSZ }\end{array}$ & $\begin{array}{l}\text { Gas seeps, } \\
\text { pockmarks }\end{array}$ & {$[158]$} \\
\hline $\begin{array}{l}\text { Swedish and } \\
\text { Norwegian (eastern } \\
\text { coast of Kattegat) }\end{array}$ & $\begin{array}{c}75- \\
160 \mathrm{~m}\end{array}$ & $\begin{aligned} \text { Width: } & 200 \mathrm{~m} \text {, length: } \\
& 1.2 \mathrm{~km}\end{aligned}$ & L. pertusa & $\begin{array}{l}\text { Shallower than } \\
\text { the GHSZ }\end{array}$ & No report & [158] \\
\hline Bill Bailey bank & $\begin{array}{c}275- \\
1020 \mathrm{~m}\end{array}$ & Height: $30 \mathrm{~m}$ & $\begin{array}{l}\text { L. pertusa } \\
\text { M. oculata }\end{array}$ & $\begin{array}{l}\text { Gas hydrates at } \\
\text { different depths }\end{array}$ & No report & {$[158]$} \\
\hline $\begin{array}{l}\text { NW Porcupine Bank } \\
\text { (Rockall Bank) }\end{array}$ & $\begin{array}{c}500- \\
1200 \mathrm{~m}\end{array}$ & $\begin{array}{l}\text { The largest: height: } \\
100 \text { m, length: } 5 \mathrm{~km}\end{array}$ & L. pertusa & $\begin{array}{c}\text { Around } \\
\text { LLGHSZ } \\
\text { Deeper than } \\
\text { the LLGHSZ }\end{array}$ & No report & {$[158]$} \\
\hline $\begin{array}{l}\text { Northernmost } \\
\text { Rockall Trough }\end{array}$ & $\begin{array}{c}900- \\
1060 \mathrm{~m}\end{array}$ & Height: $5 \mathrm{~m}$, width: $75 \mathrm{~m}$ & L. pertusa & $\begin{array}{l}\text { Deeper than } \\
\text { the LLGHSZ }\end{array}$ & Gas seeps & [115] \\
\hline $\begin{array}{l}\text { Campos Basin off } \\
\text { Brazil }\end{array}$ & $\begin{array}{c}570- \\
850 \mathrm{~m}\end{array}$ & Covers about $600 \mathrm{~km}^{2}$ & L. pertusa & $\begin{array}{l}\text { Deeper than } \\
\text { the LLGHSZ }\end{array}$ & $\begin{array}{l}\text { Hydrocarbons from } \\
\text { sediments }\end{array}$ & {$[52]$} \\
\hline Gulf of Mexico & $\begin{array}{l}400- \\
600 \mathrm{~m}\end{array}$ & $\begin{array}{c}\text { Height: } 60 \mathrm{~m} \text {, width: } \\
1.2 \mathrm{~km}\end{array}$ & L. pertusa & $\begin{array}{l}\text { Around } \\
\text { LLGHSZ }\end{array}$ & $\begin{array}{l}\text { Salt diapirs or salt } \\
\text { stocks }\end{array}$ & [158] \\
\hline Blake Ridge & $700 \mathrm{~m}$ & $\begin{array}{l}\text { Height: } 160 \mathrm{~m} \text {, width: } \\
800 \mathrm{~m}\end{array}$ & No report & $\begin{array}{l}\text { Deeper than } \\
\text { the LLGHSZ }\end{array}$ & $\begin{array}{l}\text { Gas seeps, } \\
\text { pockmark }\end{array}$ & {$[158]$} \\
\hline $\begin{array}{l}\text { Canada (east } \\
\text { Newfoundland) }\end{array}$ & $\begin{array}{c}1900- \\
2100 \mathrm{~m}\end{array}$ & $\begin{array}{l}\text { Height: } 200 \mathrm{~m} \text {, width: } \\
2 \mathrm{~km}\end{array}$ & No report & $\begin{array}{l}\text { Deeper than } \\
\text { the LLGHSZ }\end{array}$ & No report & {$[158]$} \\
\hline $\begin{array}{l}\text { South of the Charlie } \\
\text { Gibbs Fracture Zone }\end{array}$ & $1340 \mathrm{~m}$ & Diameter: $<0.5 \mathrm{~m}$ & L. pertusa & $\begin{array}{l}\text { Deeper than } \\
\text { the LLGHSZ }\end{array}$ & Gas seeps & {$[158]$} \\
\hline
\end{tabular}


TABLE 2: Continued.

\begin{tabular}{|c|c|c|c|c|c|c|}
\hline $\begin{array}{l}\text { Deep-water coral } \\
\text { location }\end{array}$ & $\begin{array}{l}\text { Water } \\
\text { depth }\end{array}$ & Size & Types & $\begin{array}{c}\text { Relationship } \\
\text { with gas } \\
\text { hydrate system }\end{array}$ & $\begin{array}{l}\text { Related fluid } \\
\text { seepage }\end{array}$ & References \\
\hline Lower Congo Basin & $400 \mathrm{~m}$ & $\begin{array}{c}\text { Height: } 30 \mathrm{~m} \text {, width: } 200- \\
400 \mathrm{~m}\end{array}$ & No report & $\begin{array}{c}\text { Shallower than } \\
\text { the GHSZ }\end{array}$ & $\begin{array}{l}\text { Gas seeps, chimney, } \\
\text { salt diapir }\end{array}$ & {$[158,160]$} \\
\hline
\end{tabular}

related to hydrocarbon seeps, on which the bacteria and other microorganisms thrived and provided the deep-water corals with substantial food source. Deep-water corals were observed at the edge of pockmarks in Santos Basin with coral rubble filling the surrounding sediments of the pockmarks, and it was proposed that the pockmark-related seabed hydrocarbon seepage may have fertilized the water by increasing the concentration of nutrients which was favorable for sustaining the corals [52]. Strong currents and low sediment supply are also regarded as favorable conditions for the deep-water coral and associated benthic fauna.

Recent studies show that the formation of deep-water corals is controlled by two major factors, one is suitable hard substratum for coral colonization such as authigenic carbonates and the other one is nutrient supply provided by dynamic bottom current, seabed methane seepage, or gas hydrate systems $[120,125]$. As a result, methane seepage features at the seabed often sustain a certain density of benthic life which is comparatively flourished compared to surrounding areas $[53,126]$. In seabed methane seepage areas, the process of methane anaerobic oxidation at the subsurface forms authigenic carbonates, and released methane feeds the microorganisms which can act as food for the deep-water corals, both favorable for the formation of deep-water corals (Figure 6(a)). Additionally, gas hydrate systems may help fertilize the deep-water corals as well $[120,127]$. A case study from Porcupine Basin shows that methane released from gas hydrate dissociation, which is glacially controlled as a result of global warming, may have played a big role in the formation of deep-water coral reefs [127].

Table 2 is the compilation of published data on deepwater corals showing information on water depth, coral size, relationship with gas hydrate system, and related fluid seepage. Results show that most of the deep-water corals occur at a depth of 200-1000 m, and their locations rely largely on hard substratum. According to the case studies in Table 2, $42.5 \%$ of the deep-water corals are related to obvious fluid seepage features and $70 \%$ of the deep-water corals are located within the GHSZ scope and nearby LLGHSZ area. Generally, case studies of the pockmarks, authigenic carbonates, and deep-water corals from all over the world indicate a genetic link, with seabed methane seepage playing a key role in the formation of carbonates and deep-water corals.

3.3. Mud Volcanoes. Compared to other seabed methane seepage manifestations, submarine mud volcanoes are relatively anonymous, mysterious, and seldomly discussed, which are topographically expressed seabed edifice due to mud and fluid eruption [129]. The presence of mud volcanoes often indicates deep petroleum assets or gas hydrate systems and offers insights into diagenetic processes like mineral dissolution and transformation, degradation of organic material, and high pressure-temperature reactions [129-131]. The geometry and size of the mud volcanoes are variable in different areas, often circular or subcircular in plane view. The height may range from several meters to hundreds of meters due to the emission scale, which is mainly controlled by tectonic activity, rapid sediment loading, the presence of thick fine-grained sediments, and enriched hydrocarbon accumulation $[55,132]$. It was proposed that compression and rapid sedimentation are mainly responsible for the initiation and maintenance of mud volcanoes, while weak argillaceous sediments under compression are prone to experience mineral alteration and dehydration processes, therefore causing brittle deformation such as faulting [130]. Additionally, the preexistence of fault zones may also act as favorable condition for the formation of mud volcanoes [133]. Different from mud volcano, there is another similar feature called mud diapir which has risen from the deep sediments into shallower sediments [129] (Figure 7). When it reaches and pierces the seabed, mud volcano will occur.

Many mud volcanoes in marine environments are associated with gas hydrate systems [129] (Figure 7). The presence of mud volcanoes is capable of changing the stability conditions of gas hydrates and causing an upvaulted BSR, and the fluid migration associated with the mud volcanoes can supply methane for the formation of gas hydrates and underlying free gas zones [134, 135]. Additionally, gas hydrate dissociation in response to sea level change, global warming, or local elevated heat flow may lead to the overpressure of the pore fluid and deformation of the sedimentary overburden, which eventually cause the expulsion of mud volcanoes $[136,137]$. In these processes, fluid migration plays a key role in both gas hydrate formation and mud volcano formation.

In addition to these seabed methane seepage manifestations we introduced above, gas hydrate pingoes are also commonly observed at seabed methane seepage sites $[138,139]$. Gas hydrate pingoes, also known as gas hydrate mounds, are the result of gas hydrate growth at the very shallow subsurface sediments. Adequate gas supply along faults, gas chimneys, and other fluid migration conduits will maintain the continuous formation of gas hydrates, triggering the overlying sediments to swell and therefore causing gas hydrate pingoes. Generally, the formation of gas hydrate pingoes also depends on fluid migration which represents the plumbing system connecting the deep hydrocarbon fluids and shallower gas hydrate reservoirs. 


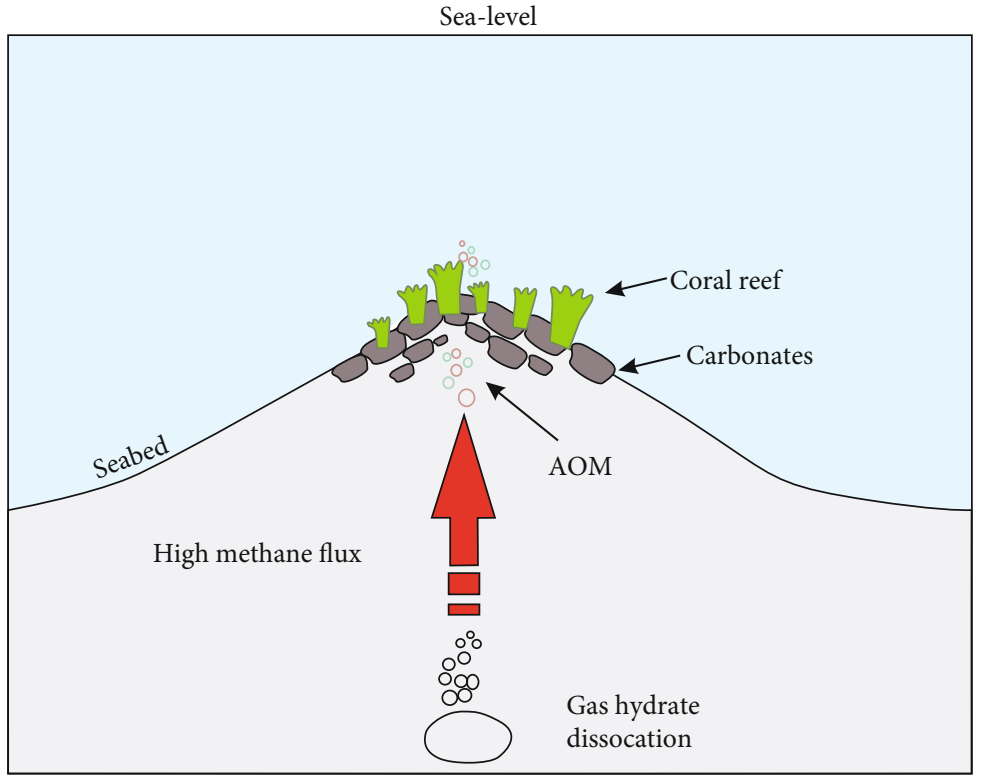

(a)

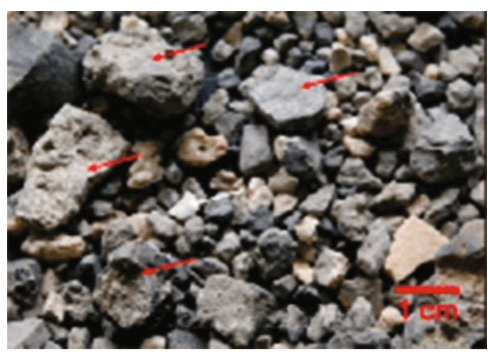

(c)

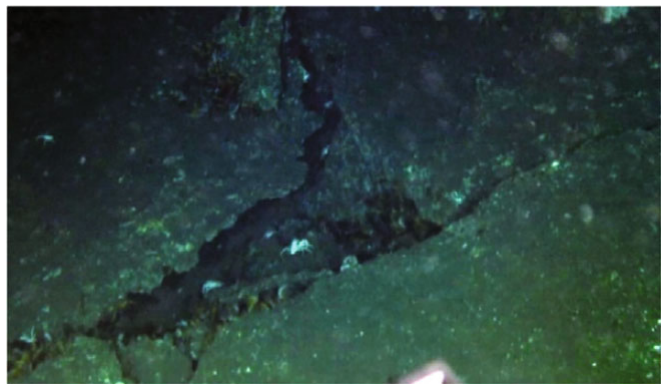

(e)

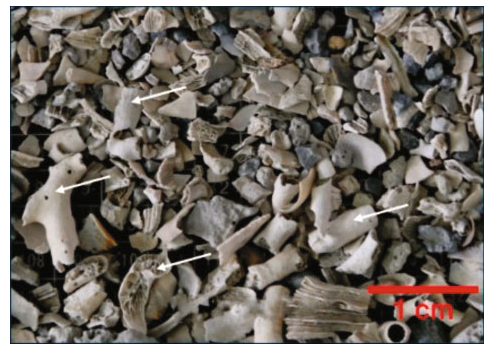

(b)

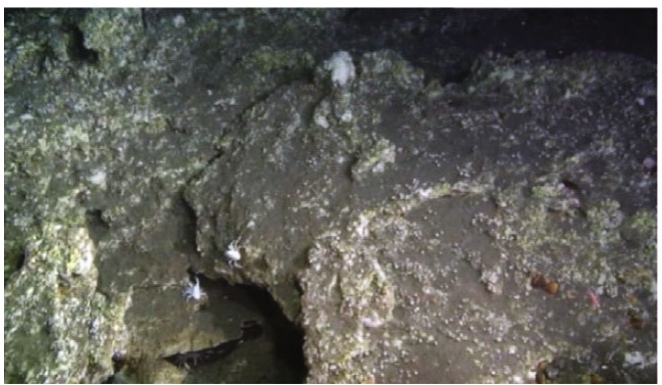

(d)

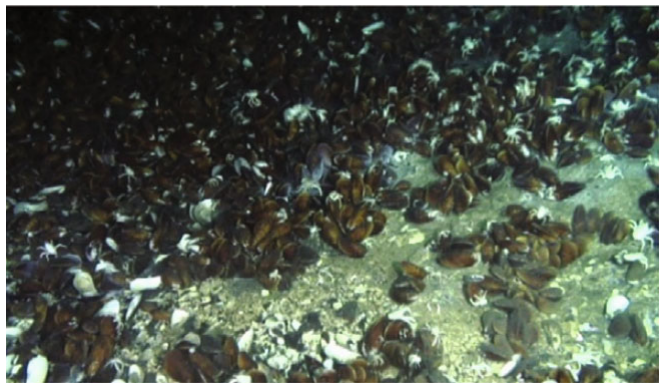

(f)

Figure 6: (a) Schematic diagram showing the formation mechanism of deep-water corals at a seabed methane seepage site. Authigenic carbonates were formed by anaerobic oxidation methane (AOM) which provided hard substratum for corals (modified from Deng et al. [120]). (b, c) Fragments of large sediments with white arrows indicating deep-water corals and red arrows indicating authigenic carbonates. (d) Large carbonate rocks at seabed methane seepage site in northern South China Sea (modified from Feng and Chen [128]). (e, f) Large carbonates and mussels suggesting that methane seepage in this area is sustainable (modified from Feng and Chen [128]).

\section{Relevant Fluid Migration Conduits}

The detailed characterization of the seabed methane seepage features shows that seabed methane seepage is often related to subsurface focused fluid migration conduits such as faults (Figure 3), gas chimneys (Figures 4, 5, and 8), unconformities (Figure 9), mud volcanoes or diapirs (Figure 7), and permeable sandstone layers. The stress change associated with factors like tectonic events or rapid sediment loading may have reactivated preexisting fractures or have triggered new fractures, therefore facilitating fluid migration $[3,12]$. The gas source of fluid migration could be from overpressured hydrocarbon reservoirs or gas hydrate systems, which can be deduced from the vertical extension of the fluid migration conduits. A seismic profile from Niger Delta shows that the gas chimney extends deeply into the deep stratum, and we propose that the gas source for the seabed methane seepage is probably from the deep hydrocarbon 


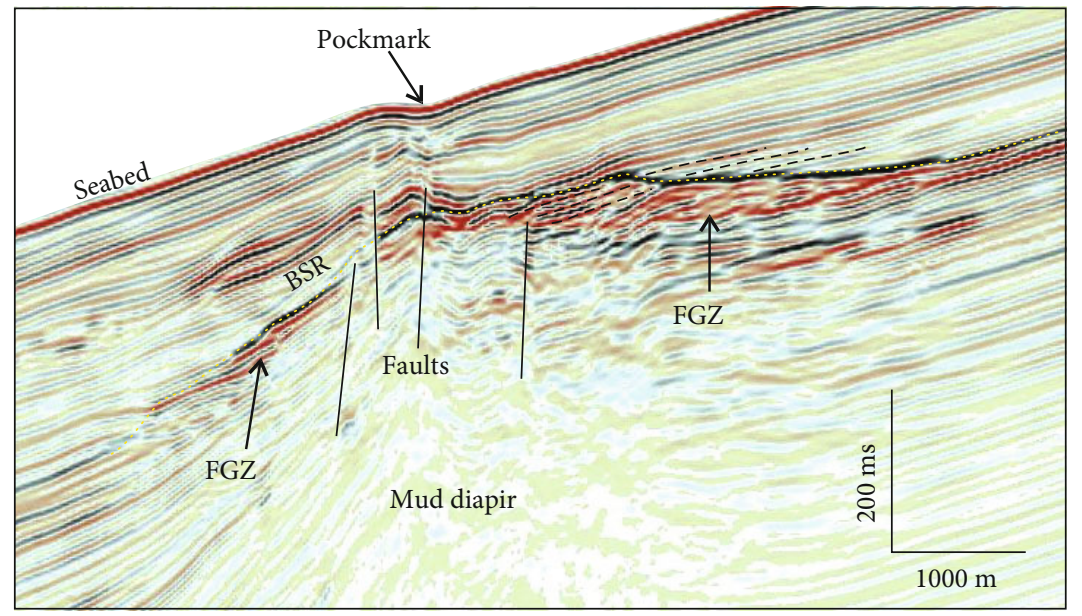

Figure 7: A seismic profile from offshore Mauritania area showing a pockmark above a mud diapir structure. The diapir structure and the overlying related faults provide an advantageous migration channel for the overpressured fluids, which formed pockmarks at the seabed. FGZ: free gas zone which is underlying the base of GHSZ.

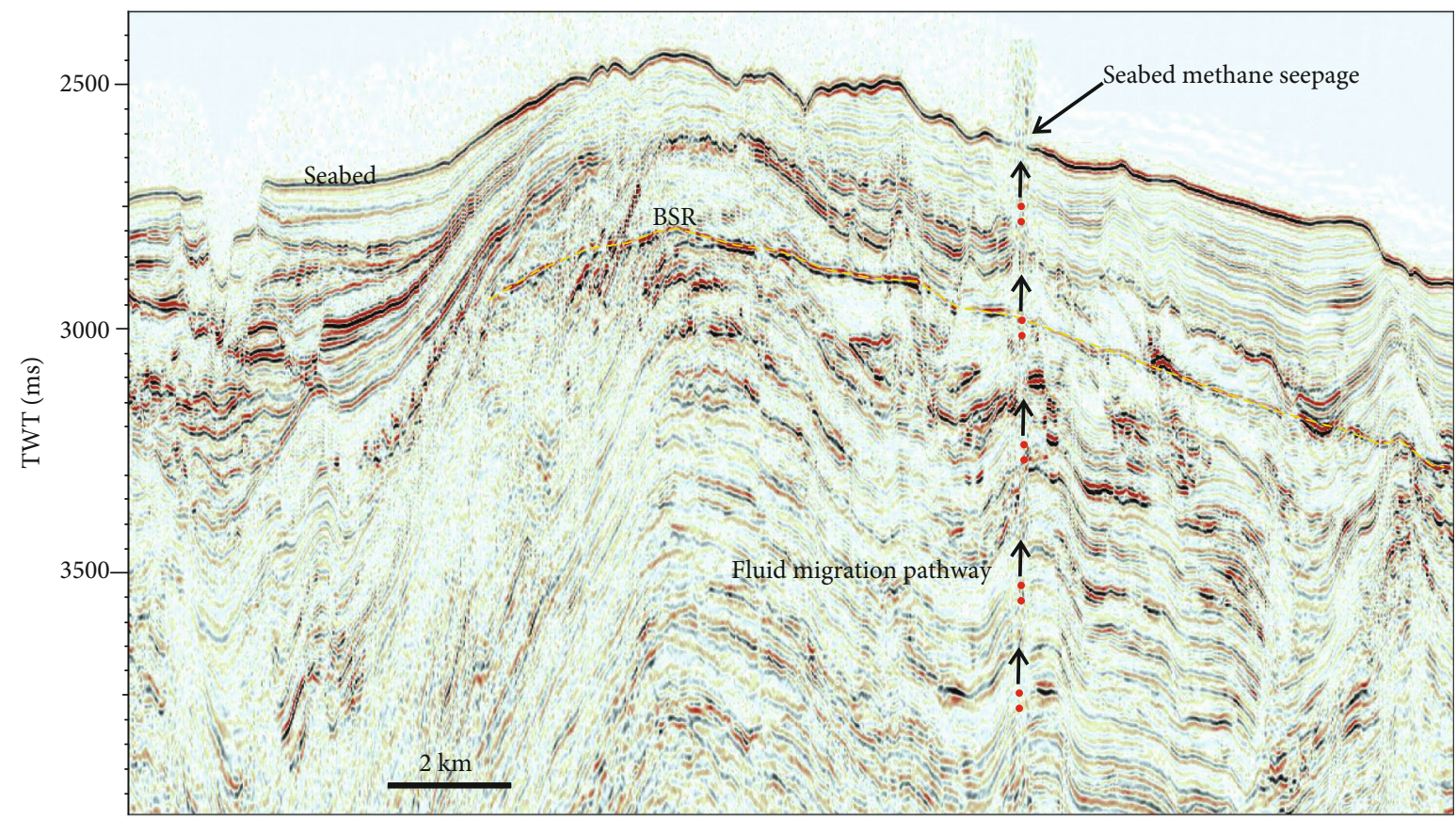

FIgURE 8: A seismic profile from Niger Delta, showing the seabed methane seepage feature and related underlying gas chimney. The gas chimney acted as fluid migration conduit, transporting the deeper fluids (free gas, water, etc.) upward through the GHSZ to the seabed.

reservoir, rather than the shallower gas hydrate systems (Figure 8). However, another seismic profile from Niger Delta shows that the unconformity and faults may act as fluid conduits which have transported methane from the free gas zone (FGZ) underlying the BSR or from gas reservoirs at the deep laterally to the updip, eventually forming seabed methane seepage at the seabed (Figure 9). Besides, the base of GHSZ, as well as permeable sandstone layers, can also act as fluid migration conduits which play roles in forming the seabed methane seepage features by supplying fluid source (Figure 4(b)). According to Westbrook et al., hundreds of gas bubble plumes have been observed emanat- ing from the seabed of the West Spitsbergen continental margin, and it was proposed that the gas source is from hydrate dissociation with released methane migrated along the base of GHSZ updip to the seabed [21].

\section{Discussions}

On the basis of above characterization of the seabed methane seepage features and related fluid migration conduits, we divide the seabed methane seepage features into three types according to its spatial relationship with the landward limit of gas hydrate stability zone (LLGHSZ), deeper than 


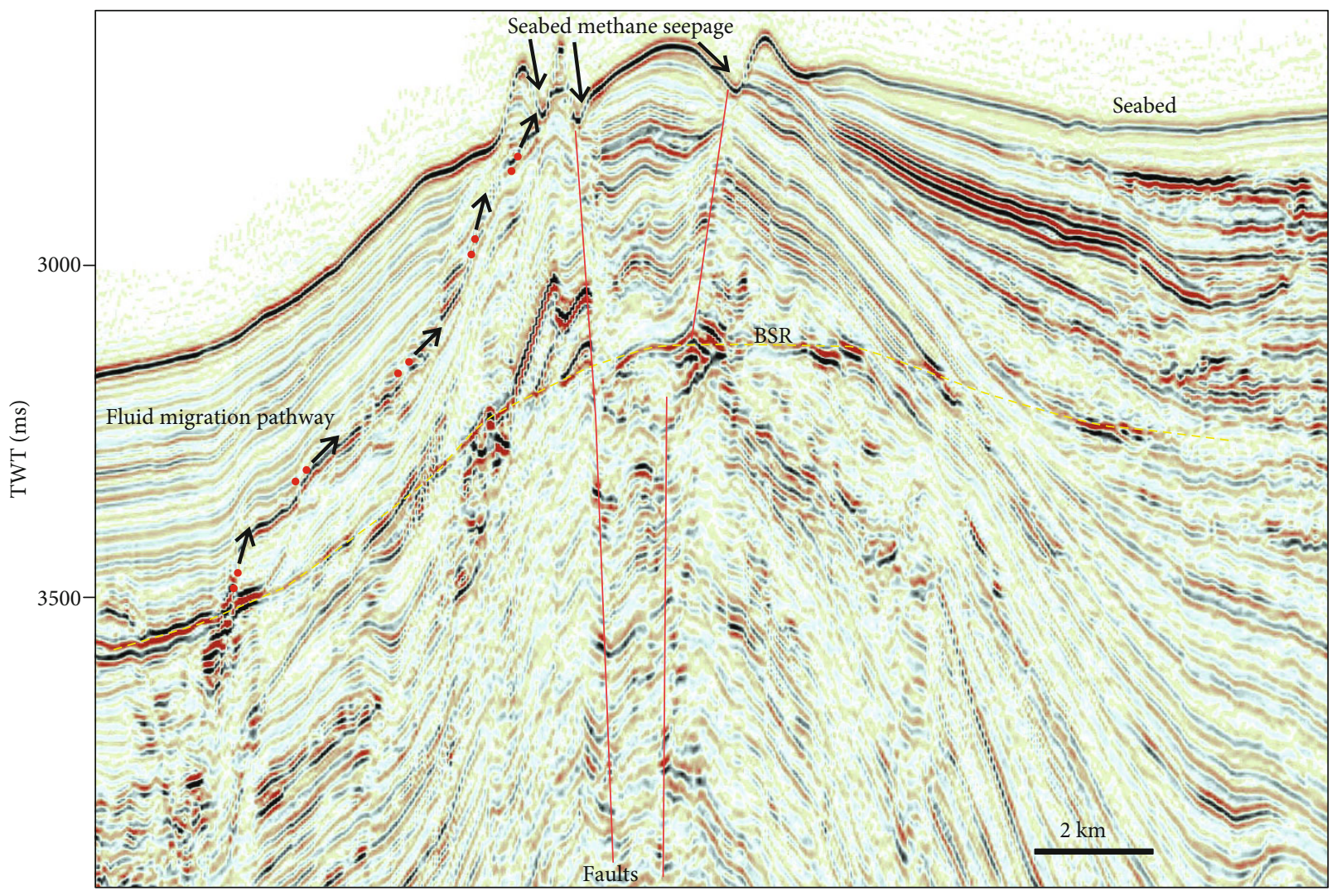

Figure 9: A seismic profile from Niger Delta, showing the unconformity surface and faults which acted as fluid migration conduits. The free gas underlying below the BSR migrated along the unconformity surface or fault to the seabed, forming methane seepage features.

TABLE 3: Relationship of GHSZ and seabed methane seepage.

Spatial relationship of seabed methane seepage and the GHSZ

Deeper than LLGHSZ A

Fault/gas chimney/diapirism/mud volcano/ unconformity surface/permeable sandstone layer/pockmark

Around LLGHSZ

B

The fluid migration conduits may be not obvious, and small-scale vertical faults/gas chimneys/pockmarks may exist

Fault/gas chimney/diapirism/mud volcano/ unconformity surface/permeable sandstone layer/pockmark C
Shallower than LLGHSZ
Methane source is irrelevant to the gas hydrate system and is from deeper gas reservoirs

Methane source is from the gas hydrate system, like gas hydrate decomposition or underlying free gas zone

Methane source is independent of the hydrate system, transported vertically along fluid migration conduits such as faults or gas chimney to the seabed

Methane source is from the gas hydrate decomposition, or methane was transported along the base of hydrate stability zone laterally to the seabed

Methane seepage sites are out of the gas hydrate stability zone, and methane source is independent of the gas hydrate system
A1 the LLGHSZ (A), around the LLGHSZ (B), and shallower than LLGHSZ (C). In plane view, the LLGHSZ is a linear feature in theory, but it often exists as a narrow zone due to the dynamic shift of the LLGHSZ in response to geological events such as glacial unloading, sea level change, and global warming [12]. These three types of seabed methane seepage can be further divided into five subtypes based on the gas source of seabed methane seepage, A1,
A2, B1, B2, and C1 (Table 3), which will be explained in the following sections.

5.1. Deeper Than LLGHSZ (A). Seabed methane seepage deeper than LLGHSZ is defined as type A in this paper, which is located in the range of GHSZ, but deeper than the landward boundary of the gas hydrate stability zone (Figures 3, 4(c), 7, 8 , and 9). Considering the gas source, type A of seabed 


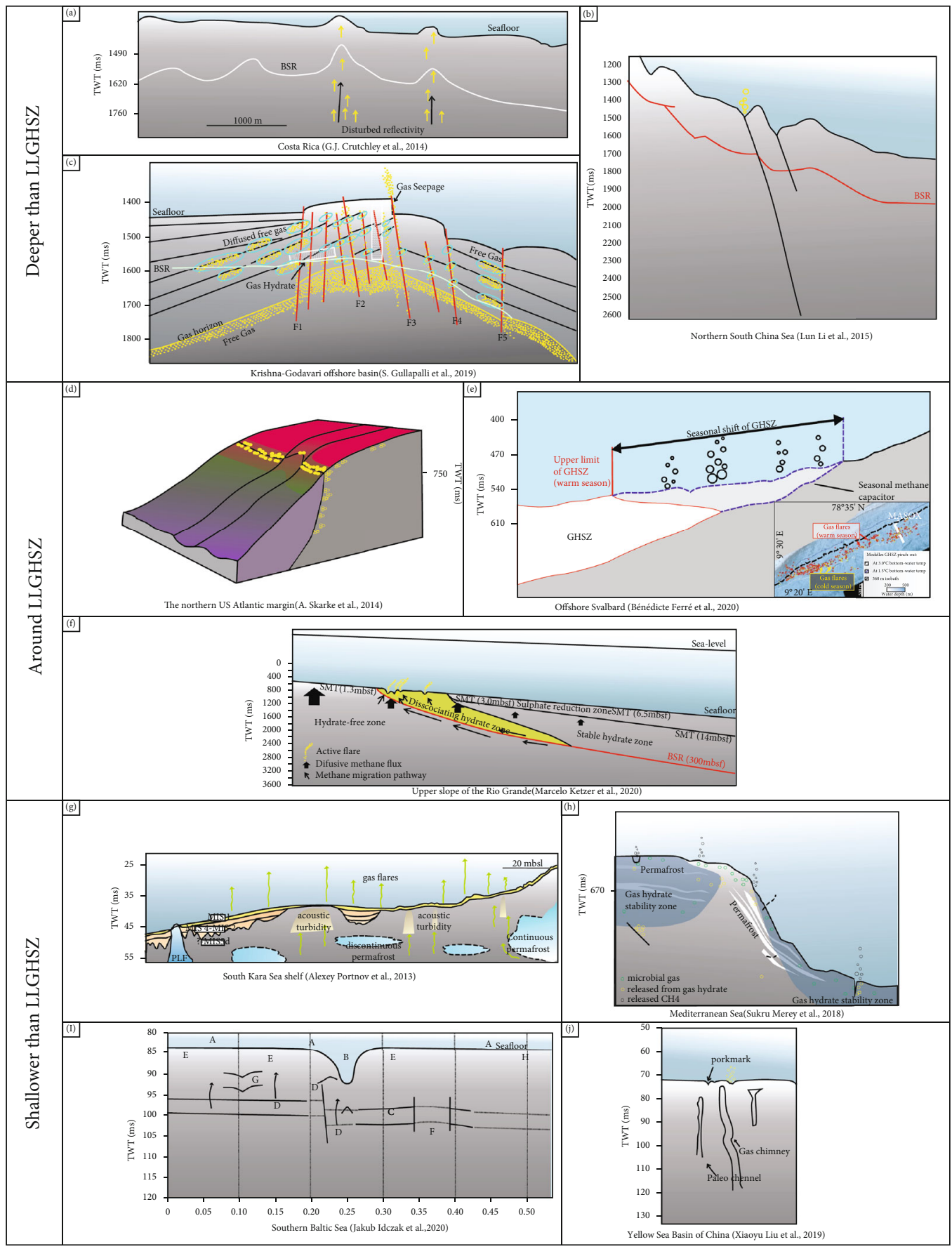

Figure 10: Compilation diagram showing different types of seabed methane seepage based on global case studies. (a) Seabed methane seepage in Costa Rica located at deeper than the LLGHSZ, with gas migrated along gas chimneys from gas reservoirs deeper than the gas hydrate systems to the seabed, defined as subtype A1 (modified from Crutchley et al. [58]). (b) Seabed methane seepage in northern South China Sea located at deeper than the LLGHSZ, with gas migrated along faults from gas reservoirs deeper than the gas hydrate systems to the seabed, defined as subtype A1 (modified from Li et al. [59]). (c) Seabed methane seepage in offshore Krishna-Godavari Basin located at deeper than the LLGHSZ, with gas migrated along faults from the gas hydrate systems to the seabed, defined as subtype A2 (modified from Gullapalli et al. [161]). (d-f) Seabed methane seepage features at around the LLGHSZ in the northern U.S. Atlantic margin, offshore Svalbard, and upper slope of the Rio Grande, defined as subtype B2. The gas sources of these methane seepage sites were all from the gas hydrate system, especially due to the gas hydrate dissociation caused by the dynamic shift of the GHSZ (modified after Skarke et al. [20]; Ferré et al. [15]; Ketzer et al. [4]). (g-j) Seabed methane seepage features at shallower than the LLGHSZ in the South Kara Sea shelf, permafrost of Mediterranean Sea, southern Baltic Sea, and Yellow Sea Basin of China, defined as subtype C1. The gas sources of these seabed methane seepage sites are irrelevant to the gas hydrate system, can be from thermogenic methane from the deep, microbial methane generated in shallow sediments, or newly released from frozen soil melting (modified from Portnov et al. [163]; Merey and Longinos [164]; Liu et al. [24]; Idczak et al. [165]). 


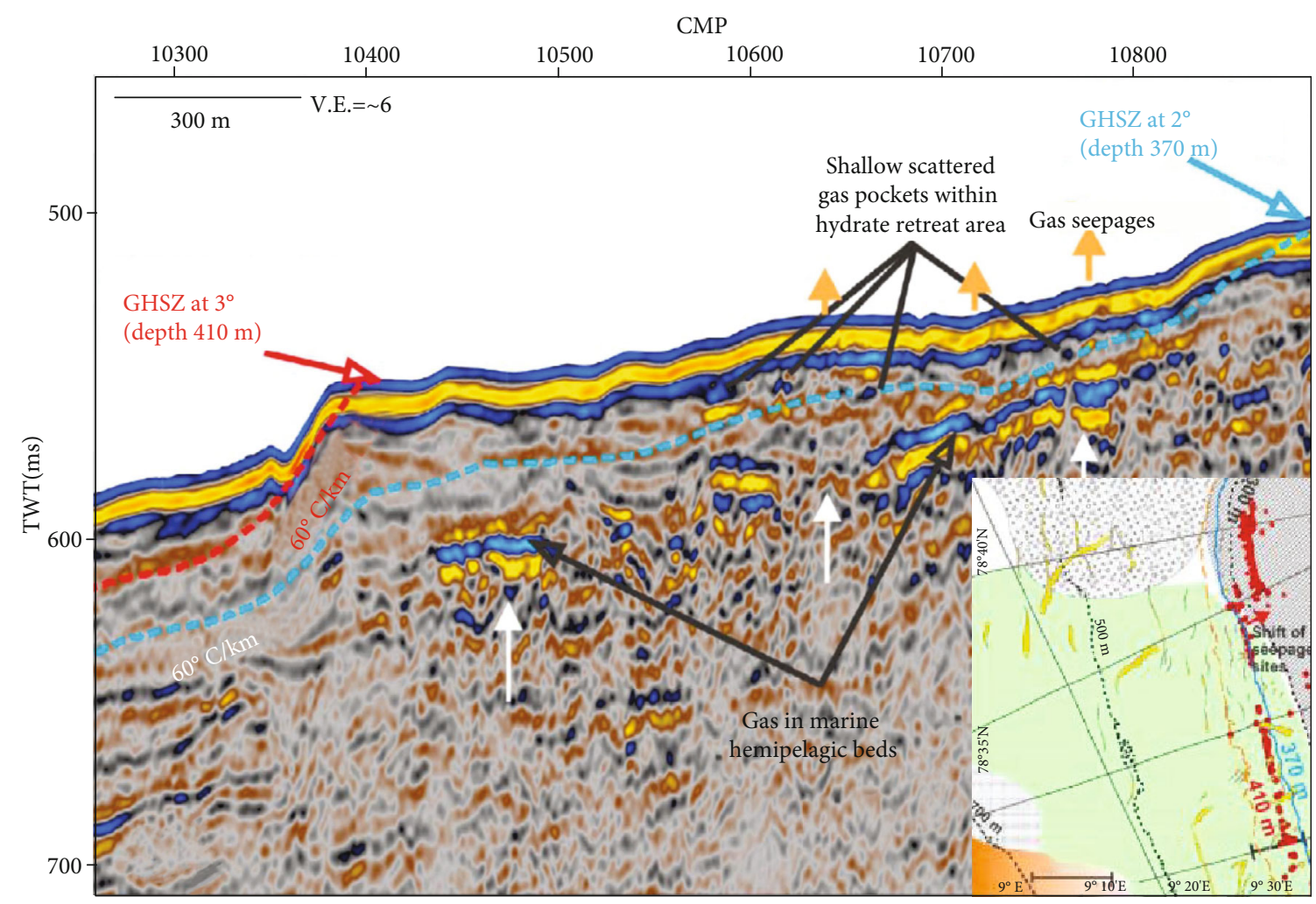

FIGURE 11: A seismic profile from offshore Svalbard, showing seabed methane seepage features at around the LLGHSZ. The LLGHSZ shifted seawards due to seasonal temperature change from $370 \mathrm{~m}$ at $2^{\circ} \mathrm{C}$ to $410 \mathrm{~m}$ at $3^{\circ} \mathrm{C}$, and related gas hydrate dissociation caused the formation of seabed methane seepage, belonging to subtype B2 (modified from Sarkar et al. [162]).

methane seepage can be further divided into two subtypes, $\mathrm{A} 1$ and A2. The methane source of A1 subtype is irrelevant to the gas hydrate systems and is probably from deeper gas reservoirs, while methane source of A2 subtype is from the gas hydrate system, either from gas hydrate decomposition or underlying free gas zone. For example, the gas source of seabed methane seepage in a case study from Costa Rica was interpreted to be from the gas reservoir which is located at deeper depth than the gas hydrate system, defined as A1 subtype [58] (Figure 10(a)). Similar seabed methane seepage features were also observed in the South China Sea where faults act as fluid migration conduits transporting gas from the gas reservoir deeper than the gas hydrate systems to the seabed, also defined as A1 subtype [59] (Figure 10(b)). In offshore Krishna-Godavari Basin, the gas source of methane seepage was interpreted to be transported along faults from the gas hydrate systems and the underlying free gas zone to the seabed, defined as A2 subtype [161] (Figure 10(c)). For both subtypes, the vertical fluid migration along focused conduits provided gas source for the formation of seabed methane seepage feature, which was probably driven by overpressure associated with tectonic compression or continuous accumulation of fluids $[18,19]$.

5.2. Around LLGHSZ (B). Seabed methane seepage located nearby the LLGHSZ is defined as type B in this paper, which is located at the landward boundary of the gas hydrate stability zone (Figures $4(\mathrm{~b}), 11,12$, and $10(\mathrm{~d})-10(\mathrm{f})$ ). The methane source of subtype B1 is defined as independent of the hydrate systems, transported vertically along fluid migration conduits such as faults or gas chimney to the seabed. Subtype B1 has rarely been observed, and most of the seabed methane seepage at the LLGHSZ is closely related to the gas hydrate systems, either from gas hydrate decomposition or methane transported laterally along the base of hydrate stability zone to the seabed which is defined as subtype B2. The gas hydrate stability zone is a wedge-shaped zone with the BSR shallowing updip as the seabed depth decreases and eventually intersecting the seabed, and the intersection location is recognized as the landward limit of the GHSZ (LLGHSZ) [47]. On the U.S. Atlantic continental margin, Skarke et al. [20] observed 570 previously undiscovered gas plumes at water depth ranging from 50 to $1700 \mathrm{mbsl}$, of which about 440 are located near the present LLGHSZ (Figure 10(d)). It was proposed that these seabed methane seepage features were caused by hydrate dissociation as a result of dynamic shift of the GHSZ due to the seabed water temperature changes, therefore defined as $\mathrm{B} 2$ subtype in this paper [20,162] (Figures 11 and 12). Similar phenomena have also been observed in offshore Svalbard (Figure 10(e)) and upper slope of the Rio Grande (Figure 10(f)), where the gas sources of seabed methane seepage were from gas hydrate dissociation [4, 15]. Many seabed pockmarks and gas plumes distributed along the linear LLGHSZ zone are caused by the periodic formation and decomposition of gas hydrate, probably due to the weak stability of gas hydrates and the variable environmental conditions at the seabed $[20,21,72]$. However, we cannot rule out another possibility 


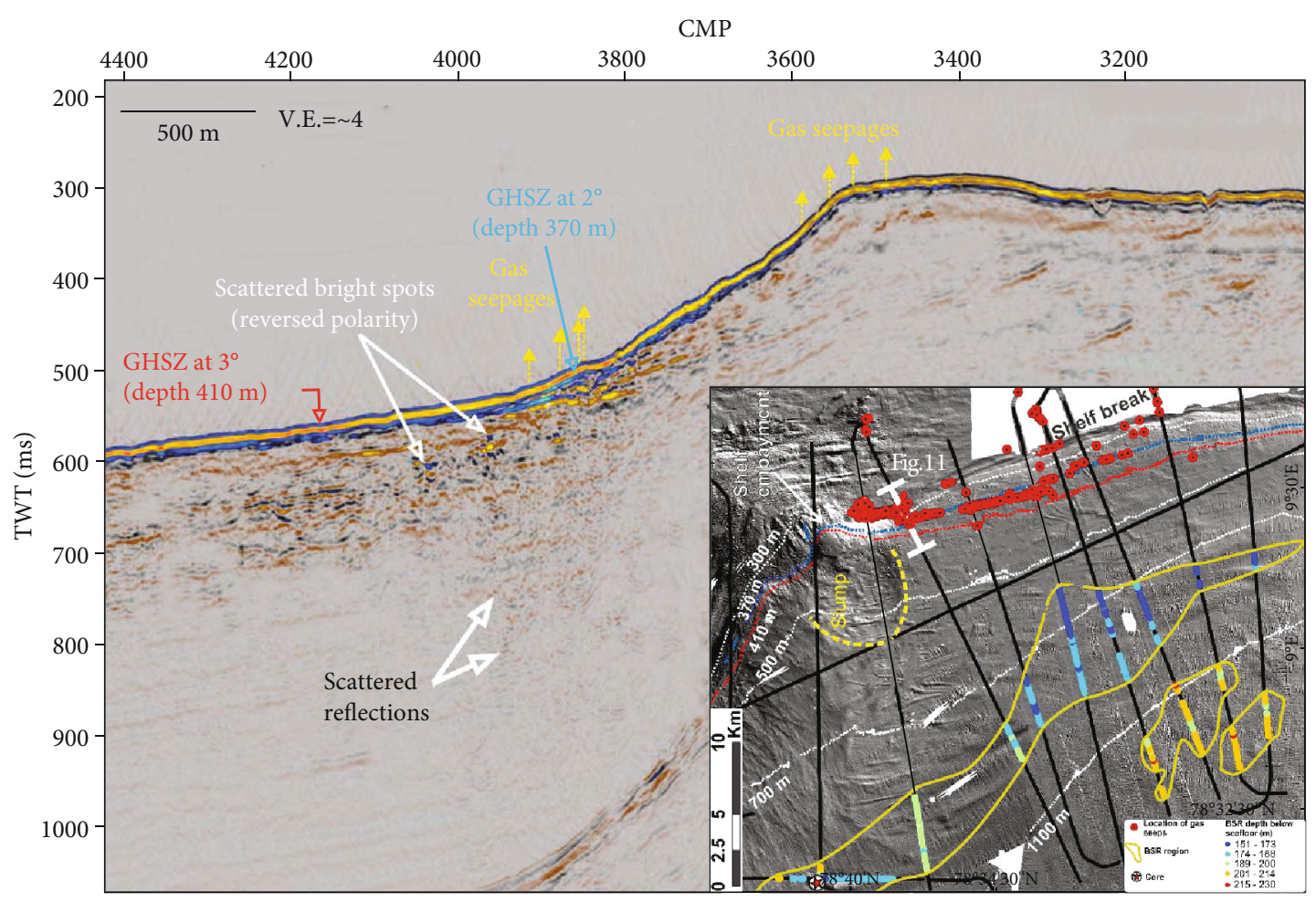

FIGURE 12: A seismic profile from offshore Svalbard, showing subtype B2 of seabed methane seepage which related to lateral migration of dissociated gas hydrates due to the water temperature increase (modified from Sarkar et al. [162]).

that at around the LLGHSZ, there may exist some seabed methane seepage features which are not related to the gas hydrate systems but transported from the deep hydrocarbon reservoirs by focused fluid conduits such as faults and chimneys, defined as B1 subtype in this study (Table 3 ).

5.3. Shallower Than LLGHSZ (C). Seabed methane seepage shallower than LLGHSZ is defined as type C in this paper, which is located outside of the range of GHSZ (Figures $10(\mathrm{~g})-10(\mathrm{j}))$. The gas source for this type of seabed methane seepage is irrelevant to the gas hydrate systems, termed as C1, can be from shallow biogenic gas or deep thermogenic gas, and was transported to the seabed through focused fluid migration conduits [24, 16-165]. For example, the seabed methane seepage on the South Kara Sea shelf has a mixed gas source which was composed of thermogenic methane from the deep and microbial methane generated in shallow sediments or newly released from frozen soil melting, belonging to $\mathrm{C} 1$ subtype (Figure $10(\mathrm{~g})$ ).

Generally, the abundant global case studies on seabed methane seepage enable us to classify the seabed methane seepage into three main types according to its spatial relationship with the LLGHSZ and gas source (Figure 13). The seabed methane seepage features manifest as gas plumes, pockmarks, authigenic carbonates, deep-water corals, and mud volcanoes and related focused fluid migration conduits include faults, gas chimneys, unconformity surfaces, and mud volcanoes. The gas source for these seabed methane seepage features can be from the gas hydrate systems including gas hydrate dissociation or the underlying free gas zone or from deeper hydrocarbon reservoirs which are irrelevant to the gas hydrate systems. Based on all these elements, the seabed methane seepage is divided into three main types, which can be further divided into five subtypes (Table 3; Figures 10-13).

Through comparison of these different seabed methane seepage types from published global case studies, we propose that subtype B2 represents the most important seabed methane seepage type. At around the landward limit of gas hydrate stability zone develop massive highly focused vigorous methane seepage features, characterized by high density of seepage sites (pockmarks or gas plumes) per kilometer and huge amounts of released methane volume [20, 21, 47, 72]. The percentage histograms of the pockmarks, carbonates, and deep-water corals in zones A, B, and C were made based on the information of global case studies shown in Tables 1 and 2, and it shows that the seabed methane seepage manifestations are prone to occur at around the LLGHSZ and shallower than the LLGHSZ (Figure 14). For example, most of the pockmarks are located at around the LLGHSZ or shallower than the LLGHSZ along the upper slope and outer shelf of the U.S. Atlantic continental margin, with limited pockmarks occurring deeper than the LLGHSZ [19]. We propose that this may be caused by the presence of GHSZ which will consume the migrated methane by forming impermeable gas hydrates, and the presence of gas hydrates also inhibits further gas migration due to its low permeability. The deep-water corals in zone B are comparatively less developed, which is probably due to the narrow width of zone B. Most of the studies on deep-water corals mainly focus on the description of the coral types and size, and limited has been done about the formation mechanism 


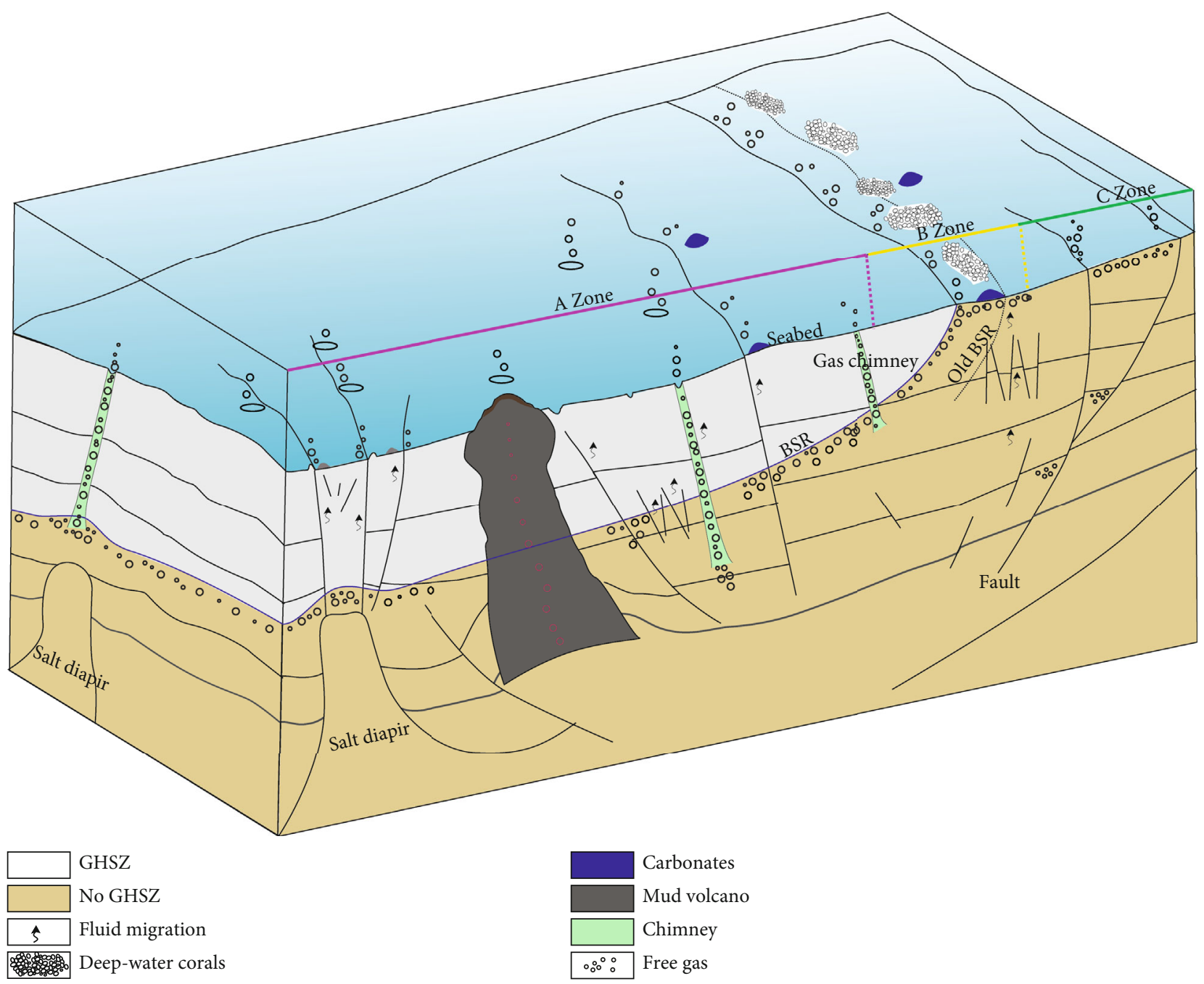

FIGURE 13: Three-dimensional model showing the three zones of different seabed methane seepage features according to the spatial relationship with the LLGHSZ and the gas source types.

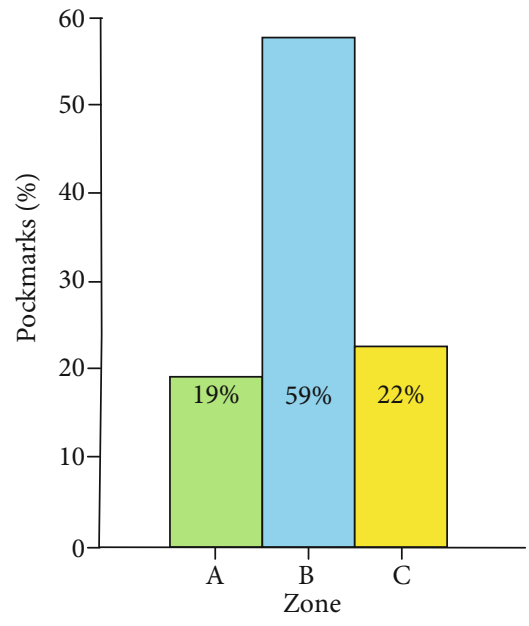

(a)

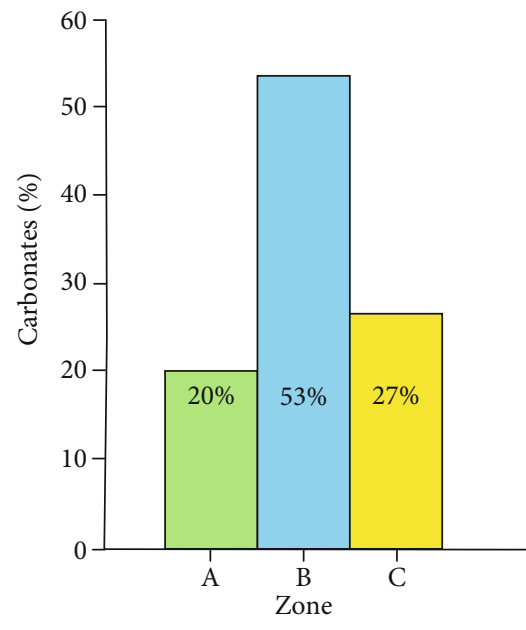

(b)

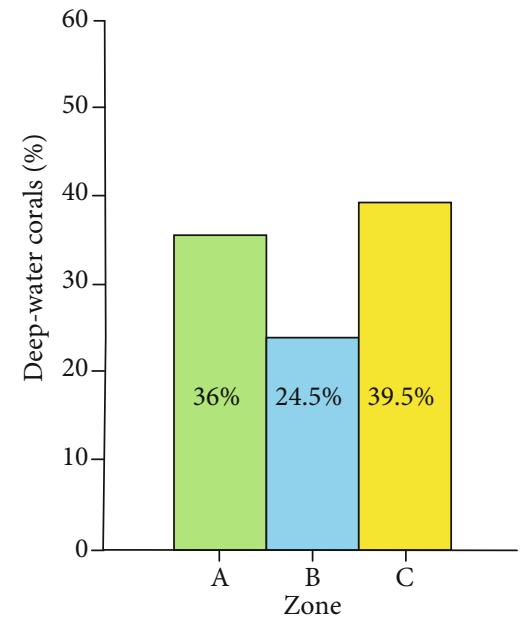

(c)

Figure 14: (a) Percentage histogram of the pockmarks in zones A, B, and C, showing that pockmarks are well developed in B zone. (b) Percentage histogram of the carbonates in zones A, B, and C, showing that carbonates are well developed in B zone. (c) Percentage histogram of the deep-water corals in zones A, B, and C, showing the distribution of deep-water corals in the three zones. 
and its relationship with the fluid migration and gas hydrate system, which may affect the representativeness of the data to certain degree.

\section{Conclusions}

A comprehensive geophysical review of the global seabed methane seepage cases has been conducted in this paper, and their relationship with the gas hydrate systems and relevant fluid migration pathways has been investigated. Compilation results show that the seabed methane seepage sites are manifested as gas plumes, pockmarks, authigenic carbonates, deep-water corals, and gas hydrate pingoes at the seabed, most of which are closely related to vertical fluid migration structures like faults, gas chimneys, mud volcanoes, and unconformity surfaces or located at the landward limit of gas hydrate stability zone (LLGHSZ). The gas source of the seabed methane seepage can be from deeper hydrocarbon reservoirs or the shallower gas hydrate systems. Considering the spatial relationship with the LLGHSZ, three major types of seabed methane seepage are classified, including deeper than the LLGHSZ (A), around the LLGHSZ (B), and shallower than LLGHSZ (C), which can be further divided into five subtypes (A1, A2, B1, B2, and C1) depending on whether the methane source is from the gas hydrate systems or not. Subtype B2 of seabed methane seepage is often characterized by high density of seepage sites and huge amounts of leaking methane, characterized by the massive focused vigorous methane seepage, which is regarded as the most important subtype probably playing a larger role in methane seepage at the seabed. Based on this research, we propose that more future measures should give priority to subtype B2 of seabed methane seepage to predict or even prevent ocean warming or climate change.

\section{Data Availability}

The data used to support the findings of this study are included within the article.

\section{Conflicts of Interest}

The authors declare that they have no conflicts of interest.

\section{Acknowledgments}

Shandong Provincial Natural Science Foundation (ZR2019QD013 and ZR201807100270), Foundation and Key Technology of Marine Gas Hydrate Trial Production Project (ZD2019-184-001), Fundamental Research Funds for the Central Universities (19CX02003A), and NSF of China (41406050) financially support this study.

\section{References}

[1] A. R. Talukder, "Review of submarine cold seep plumbing systems: leakage to seepage and venting," Terra Nova, vol. 24, no. 4, pp. 255-272, 2012.
[2] T. Pacific, "Climate change impacts, vulnerabilities and adaptation in developing countries," in United Nations Framework Convention on Climate Change, Bonn, Germany, 2017.

[3] T. Himmler, D. Sahy, T. Martma et al., “A 160,000-year-old history of tectonically controlled methane seepage in the Arctic," Science Advances, vol. 5, no. 8, p. 1450, 2019.

[4] M. Ketzer, D. Praeg, L. F. Rodrigues et al., "Gas hydrate dissociation linked to contemporary ocean warming in the southern hemisphere," Nature Communications, vol. 11, no. 1, p. 3788, 2020.

[5] E. Suess, "Marine cold seeps," Handbook of Hydrocarbon \& Lipid Microbiology, 2010.

[6] J. B. Klauda and S. I. Sandler, "Global distribution of methane hydrate in ocean sediment," Energy \& Fuels, vol. 19, no. 2, pp. 459-470, 2005.

[7] C. R. German, E. Ramirez-Llodra, M. C. Baker, P. A. Tyler, and ChEss Scientific Steering Committee, "Deep-water chemosynthetic ecosystem research during the census of marine life decade and beyond: a proposed deep-ocean road map," Plos One, vol. 6, no. 8, article 23259, 2011.

[8] J. M. Roberts, A. J. Wheeler, and A. Freiwald, "Reefs of the deep: the biology and geology of cold-water coral ecosystems," Science, vol. 312, no. 5773, pp. 543-547, 2006.

[9] T. H. Shipley, M. H. Houston, R. T. Buffler et al., "Seismic evidence for widespread possible gas hydrate horizons on continental slopes and rises," AAPG Bulletin, vol. 63, pp. 22042213, 1979.

[10] J. X. Yang, 3D Seismic Analysis of Subsurface Gas Migration and the Gas Hydrate System Offshore Mauritania, Durham University, 2013.

[11] B. J. Phrampus and M. J. Hornbach, "Recent changes to the Gulf Stream causing widespread gas hydrate destabilization," Nature, vol. 490, no. 7421, pp. 527-530, 2012.

[12] A. Crémière, A. Lepland, S. Chand et al., "Timescales of methane seepage on the Norwegian margin following collapse of the Scandinavian Ice Sheet," Nature Communications, vol. 7, no. 1, article 11509, 2016.

[13] V. Riboulot, S. Ker, N. Sultan et al., "Freshwater lake to saltwater sea causing widespread hydrate dissociation in the Black Sea," Nature Communications, vol. 9, no. 1, p. 117, 2018.

[14] G. Ciotoli, M. Procesi, G. Etiope, U. Fracassi, and G. Ventura, "Influence of tectonics on global scale distribution of geological methane emissions," Nature Communications, vol. 11, no. 1, p. 2305, 2020.

[15] B. Ferré, P. G. Jansson, M. Mooser, P. Serov, and H. Niemann, "Reduced methane seepage from Arctic sediments during cold bottom-water conditions," Nature Geoscience, vol. 13, no. 2, pp. 144-148, 2020.

[16] A. Portnov, S. Vadakkepuliyambatta, J. Mienert, and A. Hubbard, "Ice-sheet-driven methane storage and release in the Arctic," Nature Communications, vol. 7, no. 1, article 10314, 2016.

[17] C. D. Elder, D. R. Thompson, A. K. Thorpe, P. Hanke, K. M. W. Anthony, and C. E. Miller, "Airborne mapping reveals emergent power law of arctic methane emissions," Geophysical Research Letters, vol. 47, no. 3, article 58757, 2020.

[18] L. L. Brothers, C. L. van Dover, C. R. German et al., "Evidence for extensive methane venting on the southeastern U.S. Atlantic margin," Geology, vol. 41, no. 7, pp. 807-810, 2013.

[19] D. S. Brothers, C. Ruppel, J. W. Kluesner et al., "Seabed fluid expulsion along the upper slope and outer shelf of the U.S. 
Atlantic continental margin," Geophysical Research Letters, vol. 41, no. 1, pp. 96-101, 2014.

[20] A. Skarke, C. Ruppel, M. Kodis, D. Brothers, and E. Lobecker, "Widespread methane leakage from the sea floor on the northern US Atlantic margin," Nature Geoscience, vol. 7, no. 9, pp. 657-661, 2014.

[21] G. K. Westbrook, K. E. Thatcher, E. J. Rohling et al., "Escape of methane gas from the seabed along the West Spitsbergen continental margin," Geophysical Research Letters, vol. 36, article 15608, 2009.

[22] P. Serov, S. Vadakkepuliyambatta, J. Mienert et al., "Postglacial response of Arctic Ocean gas hydrates to climatic amelioration," Proceedings of the National Academy of Sciences, vol. 114, no. 24, article 19288, 2017.

[23] S. Sauer, W. L. Hong, H. Yao, A. Lepland, and J. Knies, "Methane transport and sources in an Arctic deep-water cold seep offshore NW Svalbard (Vestnesa Ridge, $79^{\circ} \mathrm{N}$ )," Deep Sea Research Part I Oceanographic Research Papers, vol. 167, article 103430, 2020.

[24] X. Y. Liu, X. L. Feng, Y. F. Sun et al., "Acoustic and biological characteristics of seafloor depressions in the North Yellow Sea Basin of China: active fluid seepage in shallow water seafloor," Marine Geology, vol. 414, pp. 34-46, 2019.

[25] E. Suess, "Marine cold seeps and their manifestations: geological control, biogeochemical criteria and environmental conditions," International Journal of Earth Sciences, vol. 103, no. 7, pp. 1889-1916, 2014.

[26] Y. Zhang, M. Luo, Y. Hu, H. Wang, and D. Chen, “An areal assessment of subseafloor carbon cycling in cold seeps and hydrate- bearing areas in the northern South China Sea," Geofluids, vol. 2019, 14 pages, 2019.

[27] D. W. Schoell and R. von Huene, "Crustal recycling at modern subduction zones applied to the past-issues of growth and preservation of continental basement crust, mantle geochemistry, and supercontinent reconstruction," Memoir of the Geological Society of America, vol. 200, pp. 9-32, 2007.

[28] A. Boetius and F. Wenzhöfer, "Seafloor oxygen consumption fuelled by methane from cold seeps," Nature Geoscience, vol. 6, pp. 725-734, 2013.

[29] V. Riboulot, Y. Thomas, S. Berné, G. Jouet, and A. Cattaneo, "Control of Quaternary sea-level changes on gas seeps," Geophysical Research Letters, vol. 41, no. 14, pp. 4970-4977, 2014.

[30] M. Bonini, "Seismic loading of fault-controlled fuid seepage systems by great subduction earthquakes," Scientific Reports, vol. 9, article 11332, 2019.

[31] I. Leifer, B. P. Luyendyk, J. Boles, and J. F. Clark, "Natural marine seepage blowout: contribution to atmospheric methane," Global Biogeochemical Cycles, vol. 20, no. 3, 2006.

[32] I. Leifer, "Seabed bubble flux estimation by calibrated video survey for a large blowout seep in the North Sea," Marine \& Petroleum Geology, vol. 68, pp. 743-752, 2015.

[33] H. Doust and E. Omatsola, Niger Delta, Divergent/Passive Margin Basins, J. D. Edwards, P. A, Santogrossi, 1989.

[34] H. A. Cohen and K. McClay, "Sedimentation and shale tectonics of the northwestern Niger Delta front," Marine and Petroleum Geology, vol. 13, no. 3, pp. 313-328, 1996.

[35] R. J. Davies, J. Yang, A. Li, S. Mathias, and R. Hobbs, “An irregular feather-edge and potential outcrop of marine gas hydrate along the Mauritanian margin," Earth \& Planetary Science Letters, vol. 423, pp. 202-209, 2015.
[36] R. J. Davies, M. Á. Morales Maqueda, A. Li, and A. Ganopolski, "Millennial-scale shifts in the methane hydrate stability zone due to Quaternary climate change," Geology, vol. 45, no. 11, pp. 1027-1030, 2017.

[37] W. Zhang, J. Liang, J. Lu et al., “Accumulation features and mechanisms of high saturation natural gas hydrate in Shenhu Area, northern South China Sea," Shiyou Kantan Yu Kaifa/Petroleum Exploration and Development, vol. 44, no. 5, pp. 708-719, 2017.

[38] J. Q. Liang, W. Zhang, J. A. Lu, J. Wei, Z. Kuang, and Y. He, "Geological occurrence and accumulation mechanism of natural gas hydrates in the eastern Qiongdongnan Basin of the South China Sea: insights from site GMGS5-W9-2018," Marine Geology, vol. 418, article 106042, 2019.

[39] A. V. Milkov and R. Sassen, "Estimate of gas hydrate resource, northwestern Gulf of Mexico continental slope," Marine Geology, vol. 179, no. 1-2, pp. 71-83, 2001.

[40] C. K. Paull and W. P. Dillon, Natural Gas Hydrates: Occurrence, Distribution, and Detection, American Geophysical Union, 2001.

[41] G. Bohrmann, J. Greinert, E. Suess, and M. Torres, "Authigenic carbonates from the Cascadia subduction zone and their relation to gas hydrate stability," Geology, vol. 26, no. 7, pp. 647-650, 1998.

[42] J. Greinert, G. Bohrmann, and E. Suess, "Gas hydrateassociated carbonates and methane-venting at hydrate ridge: classification, distribution, and origin of authigenic lithologies," Natural Gas Hydrates Occurrence Distribution \& Detection, vol. 124, pp. 99-113, 2001.

[43] R. J. Davies, J. Yang, R. Hobbs, and A. Li, "Probable patterns of gas flow and hydrate accretion at the base of the hydrate stability zone," Geology, vol. 42, no. 12, pp. 1055-1058, 2014.

[44] J. I. T. Hillman, A. E. Cook, D. E. Sawyer, H. M. Küçük, and D. S. Goldberg, "The character and amplitude of 'discontinuous' bottom-simulating reflections in marine seismic data," Earth and Planetary Science Letters, vol. 459, pp. 157-169, 2017.

[45] S. Ker, Y. Thomas, V. Riboulot et al., "Anomalously deep BSR related to a transient state of the gas hydrate system in the western Black Sea," Geochemistry Geophysics Geosystems, vol. 20, no. 1, pp. 442-459, 2019.

[46] J. Mienert, M. Vanneste, S. Bünz, K. Andreassen, H. Haflidason, and H. P. Sejrup, "Ocean warming and gas hydrate stability on the mid-Norwegian margin at the Storegga Slide," Marine and Petroleum Geology, vol. 22, no. 1-2, pp. 233-244, 2005.

[47] C. A. Graves, L. Steinle, G. Rehder et al., "Fluxes and fate of dissolved methane released at the seafloor at the landward limit of the gas hydrate stability zone offshore western Svalbard," Journal of Geophysical Research Oceans, vol. 120, no. 9, pp. 6185-6201, 2015.

[48] C. A. Graves, R. H. James, and C. J. Sapart, "Methane in shallow subsurface sediments at the landward limit of the gas hydrate stability zone offshore western Svalbard," Geochimica et Cosmochimica Acta, vol. 198, pp. 419-438, 2017.

[49] K. U. Hinrichs, J. M. Hayes, S. P. Sylva, P. G. Brewer, and E. F. Delong, "Methane-consuming archaebacteria in marine sediments," Nature, vol. 398, no. 6730, pp. 802-805, 1999.

[50] R. Luff, J. Greinert, K. Wallmann, I. Klaucke, and E. Suess, "Simulation of long-term feedbacks from authigenic 
carbonate crust formation at cold vent sites," Chemical Geology, vol. 216, no. 1-2, pp. 157-174, 2005.

[51] G. J. Moridis, "Numerical studies of gas production from methane hydrates," SPE Journal, vol. 8, no. 4, pp. 359-370, 2003.

[52] P. Y. G. Sumida, M. Y. Yoshinaga, L. A. S. P. Madureira, and M. Hovland, "Seabed pockmarks associated with deepwater corals off SE Brazilian continental slope, Santos Basin," Marine Geology, vol. 207, no. 1-4, pp. 159-167, 2004.

[53] G. Panieri, S. Bunz, and J. E. Johnson, "An integrated view of the methane system in the pockmarks at Vestnesa Ridge, $79^{\circ} \mathrm{N}, "$ Marine Geology, vol. 390, pp. 282-300, 2017.

[54] M. Hovland and A. G. Judd, Seabed Pockmark and Seepage: Impact on Geology, Biology and the Marine Environment, 1988.

[55] A. Judd and M. Hovland, Seabed Fluid Flow-Impact on Geology, Biology and the Marine Environment, Cambridge University Press, 2007.

[56] J. Cartwright and C. Santamarina, "Seismic characteristics of fluid escape pipes in sedimentary basins: implications for pipe genesis," Marine \& Petroleum Geology, vol. 65, pp. 126-140, 2015.

[57] M. Foschi, J. Cartwright, C. W. Macminn, and G. Etiope, "Evidence for massive emission of methane from a deep-water gas field during the Pliocene," Proceedings of the National Academy of Sciences, vol. 117, no. 45, pp. 27869-27876, 2020.

[58] G. J. Crutchley, D. Klaeschen, L. Planert et al., "The impact of fluid advection on gas hydrate stability: investigations at sites of methane seepage offshore Costa Rica," Earth \& Planetary Science Letters, vol. 401, pp. 95-109, 2014.

[59] L. Li, H. Liu, X. Zhang, X. Lei, and Z. Sha, "BSRs, estimated heat flow, hydrate-related gas volume and their implications for methane seepage and gas hydrate in the Dongsha region, northern South China Sea," Marine \& Petroleum Geology, vol. 67, pp. 785-794, 2015.

[60] A. Plaza-Faverola, S. Bünz, J. E. Johnson et al., "Role of tectonic stress in seepage evolution along the gas hydratecharged Vestnesa Ridge, Fram Strait," Geophysical Research Letters, vol. 42, no. 3, pp. 733-742, 2015.

[61] T. Velayatham, S. P. Holford, and M. A. Bunch, "Ancient fluid flow recorded by remarkably long, buried pockmark trains observed in 3D seismic data, Exmouth Plateau, Northern Carnarvon Basin," Marine \& Petroleum Geology, vol. 95, pp. 303-313, 2018.

[62] H. H. Roberts, D. Feng, and S. B. Joye, "Cold-seep carbonates of the middle and lower continental slope, northern Gulf of Mexico," Deep Sea Research Part II Topical Studies in Oceanography, vol. 57, no. 21-23, pp. 2040-2054, 2010.

[63] D. Oppo, I. Viola, and R. Capozzi, "Fluid sources and stable isotope signatures in authigenic carbonates from the Northern Apennines, Italy," Marine \& Petroleum Geology, vol. 86, pp. 606-619, 2017.

[64] C. Cassarini, E. R. Rene, S. Bhattarai, C. Vogt, N. Musat, and P. N. L. Lens, "Anaerobic methane oxidation coupled to sulfate reduction in a biotrickling filter: reactor performance and microbial community analysis," Chemosphere, vol. 236, article 124290, 2019.

[65] S. Ritger, B. Carson, and E. Suess, "Methane-derived authigenic carbonates formed by subduction-induced pore-water expulsion along the Oregon/Washington margin," Geological Society of America Bulletin, vol. 98, pp. 147-156, 1987.
[66] J. Peckmann, V. Thiel, W. Michaelis et al., "Cold seep deposits of Beauvoisin (Oxfordian; southeastern France) and Marmorito (Miocene; northern Italy): microbially induced authigenic carbonates," International Journal of Earth Sciences, vol. 88, no. 1, pp. 60-75, 1999.

[67] M. Hovland, "On the self-sealing nature of marine seeps," Continental Shelf Research, vol. 22, pp. 2387-2394, 2002.

[68] J. Greinert, K. B. Lewis, J. Bialas et al., "Methane seepage along the Hikurangi Margin, New Zealand: overview of studies in 2006 and 2007 and new evidence from visual, bathymetric and hydroacoustic investigations," Marine Geology, vol. 272, no. 1-4, pp. 6-25, 2010.

[69] F. Chen, X. Wang, N. Li et al., "Gas hydrate dissociation during sea-level highstand inferred from U/Th dating of seep carbonate from the South China Sea," Geophysical Research Letters, vol. 46, no. 23, pp. 13928-13938, 2019.

[70] M. Hovland, H. Svensen, C. F. Forsberg et al., "Complex pockmarks with carbonate-ridges off mid-Norway: products of sediment degassing," Marine Geology, vol. 218, no. 1-4, pp. 191-206, 2005.

[71] C. K. Paull and W. Ussler III, "Re-evaluating the significance of seafloor accumulations of methane-derived carbonates: seepage or erosion indicators?," in International Conference on Gas Hydrates (ICGH), Vancouver, British Columbia, Canada, 2008.

[72] C. Berndt, T. Feseker, T. Treude et al., "Temporal constraints on hydrate-controlled methane seepage off Svalbard," Science, vol. 343, no. 6168, pp. 284-287, 2014.

[73] K. Andreassen, A. Hubbard, M. Winsborrow et al., "Massive blow-out craters formed by hydrate-controlled methane expulsion from the Arctic seafloor," Science, vol. 356, no. 6341, pp. 948-953, 2017.

[74] P. R. Vogt, K. Crane, E. Sundvor, M. D. Max, and S. L. Pfirman, "Methane-generated(?) pockmarks on young, thickly sedimented oceanic crust in the Arctic: Vestnesa ridge, Fram strait," Geology, vol. 22, no. 3, p. 255, 1994.

[75] S. Bünz, S. Polyanov, S. Vadakkepuliyambatta, C. Consolaro, and J. Mienert, "Active gas venting through hydrate-bearing sediments on the Vestnesa Ridge, offshore W-Svalbard," Marine Geology, vol. 332-334, pp. 189-197, 2012.

[76] M. Forwick, N. J. Baeten, and T. O. Vorren, "Pockmarks in Spitsbergenfjords," Norwegian Journal of Geology, vol. 89, pp. 65-77, 2009.

[77] A. Solheim and A. Elverhøi, "A pockmark field in the Central Barents Sea; gas from a petrogenic source?," Polar Research, vol. 3, no. 1, pp. 11-19, 1985.

[78] S. Chand, L. Rise, D. Ottesen, M. F. J. Dolan, V. Bellec, and R. Bøe, "Pockmark-like depressions near the Goliat hydrocarbon field, Barents Sea: morphology and genesis," Marine and Petroleum Geology, vol. 26, no. 7, pp. 10351042, 2009.

[79] M. Pau, G. Gisler, and Ø. Hammer, "Experimental investigation of the hydrodynamics in pockmarks using particle tracking velocimetry," Geo-Marine Letters, vol. 34, no. 1, pp. 1119, 2014.

[80] M. Pau, Ø. Hammer, and S. Chand, "Constraints on the dynamics of pockmarks in the SW Barents Sea: evidence from gravity coring and high-resolution, shallow seismic profiles," Marine Geology, vol. 355, pp. 330-345, 2014.

[81] M. A. Abrams, "Geophysical and geochemical evidence for subsurface hydrocarbon leakage in the Bering Sea, Alaska," 
Marine and Petroleum Geology, vol. 9, no. 2, pp. 208-221, 1992.

[82] A. Mazzini, H. H. Svensen, and C. F. Forsberg, “A climatic trigger for the giant Troll pockmark field in the northern North Sea," Earth and Planetary Science Letters, vol. 464, pp. 24-34, 2017.

[83] M. Hovland, R. Heggland, M. H. De Vries, and T. I. Tjelta, "Unit-pockmarks and their potential significance for predicting fluid flow," Marine and Petroleum Geology, vol. 27, pp. 1190-1199, 2010.

[84] A. Plaza-Faverola, S. Bünz, and J. Mienert, "Fluid distributions inferred from $\mathrm{P}$-wave velocity and reflection seismic amplitude anomalies beneath the Nyegga pockmark field of the mid-Norwegian margin," Marine and Petroleum Geology, vol. 27, no. 1, pp. 46-60, 2010.

[85] J. Mienert, J. Posewang, and M. Baumann, "Gas hydrates along the northeastern Atlantic margin: possible hydratebound margin instabilities and possible release of methane," Geological Society London Special Publications, vol. 137, no. 1, pp. 275-291, 1998.

[86] M. Hovland, "Large pockmarks, gas-charged sediments and possible clay diapirs in the Skagerrak," Marine and Petroleum Geology, vol. 8, no. 3, pp. 311-316, 1991.

[87] E. J. Halliday, J. V. Barrie, N. R. Chapman, and K. M. M. Rohr, "Structurally controlled hydrocarbon seeps on a glaciated continental margin, Hecate Strait, offshore British Columbia," Marine Geology, vol. 252, no. 3-4, pp. 193-206, 2008.

[88] J. V. Barrie, S. Cook, and K. W. Conway, "Cold seeps and benthic habitat on the Pacific margin of Canada," Continental Shelf Research, vol. 31, no. 2, pp. S85-S92, 2011.

[89] D. Long, "Devensian late-glacial gas escape in the central North Sea," Continental Shelf Research, vol. 12, pp. 10971110, 1992.

[90] J. T. Kelley, S. M. Dickson, D. F. Belknap, W. A. Barnhardt, and M. Henderson, "Giant sea-bed pockmarks: evidence for gas escape from Belfast Bay, Maine," Maine Geology, vol. 22, no. 1, pp. 59-62, 1994.

[91] C. Paull, W. Ussler III, and N. Maher, "Pockmarks off Big Sur, California," Marine Geology, vol. 181, no. 4, pp. 323-335, 2002.

[92] R. Sassen, S. Losh, L. Cathles et al., "Massive vein-filling gas hydrate: relation to ongoing gas migration from the deep subsurface in the Gulf of Mexico," Marine and Petroleum Geology, vol. 18, no. 5, pp. 551-560, 2001.

[93] M. M. De Mahiques, U. Schattner, and M. Lazar, "An extensive pockmark field on the upper Atlantic margin of Southeast Brazil: spatial analysis and its relationship with salt diapirism," Heliyon, vol. 3, no. 2, article e00257, 2017.

[94] N. Sultan, B. Marsset, S. Ker et al., "Hydrate dissolution as a potential mechanism for pockmark formation in the Niger Delta," Journal of Geophysical Research, vol. 115, no. B8, article B08101, 2010.

[95] Y. Marcon, H. Ondréas, H. Sahling, G. Bohrmann, and K. Olu, "Fluid flow regimes and growth of a giant pockmark," Geology, vol. 42, no. 1, pp. 63-66, 2014.

[96] H. Sahling, G. Bohrmann, V. Spiess et al., "Pockmarks in the northern Congo fan area, SW Africa: complex seafloor features shaped by fluid flow," Marime Geology, vol. 249, no. 3-4, pp. 206-225, 2008.

[97] S. Wenau, V. Spieß, T. Pape, and N. Fekete, "Controlling mechanisms of giant deep water pockmarks in the Lower
Congo Basin," Marine and Petroleum Geology, vol. 83, pp. 140-157, 2017.

[98] J. Chen, H. Song, Y. Guan et al., "Morphologies, classification and genesis of pockmarks, mud volcanoes and associated fluid escape features in the northern Zhongjiannan Basin, South China Sea," Deep-Sea Research II, vol. 122, pp. 106$117,2015$.

[99] Q. Sun, S. Wu, M. Hovland, P. Luo, Y. Lu, and T. Qu, "The morphologies and genesis of mega-pockmarks near the Xisha Uplift, South China Sea," Marine and Petroleum Geology, vol. 28, no. 6, pp. 1146-1156, 2011.

[100] D. Casas, G. Ercilla, and J. Baraza, “Acoustic evidences of gas in the continental slope sediments of the Gulf of Cadiz (E Atlantic)," Geo-Marine Letters, vol. 23, pp. 300-310, 2003.

[101] M. Ingrassia, E. Martorelli, A. Bosman, L. Macelloni, A. Sposato, and F. L. Chiocci, "The Zannone Giant Pockmark: first evidence of a giant complex seeping structure in shallow-water, central Mediterranean Sea, Italy," Marine Geology, vol. 363, pp. 38-51, 2015.

[102] L. Somoza, V. Diaz-del-Rio, R. León et al., "Seabed morphology and hydrocarbon seepage in the Gulf of Cadiz mud volcano area: acoustic imagery, multibeam and ultra-high resolution seismic data," Marine Geology, vol. 195, no. 1-4, pp. 153-176, 2003.

[103] J. Baraza and G. Ercilla, "Gas-charged sediments and large pockmark-like features on the Gulf of Cadiz slope (SW Spain)," Marine and Petroleum Geology, vol. 3, no. 2, pp. 253-261, 1996.

[104] T. Hasiotis, G. Papatheodorou, N. Kastanos, and G. Ferentinos, "A pockmark field in the Patras Gulf (Greece) and its activation during the 14/7/93 seismic event," Marine Geology, vol. 130, pp. 333-344, 1996.

[105] D. Christodoulou, G. Papatheodorou, G. Ferentinos, and M. Masson, "Active seepage in two contrasting pockmark fields in the Patras and Corinth gulfs, Greece," Geo-Marine Letters, vol. 23, pp. 194-199, 2003.

[106] A. García-García, D. L. Orange, N. M. Maher, A. S. Heffernan, G. S. Fortier, and A. Malone, "Geophysical evidence for gas geohazards off Iskenderun Bay, SE Turkey," Marine and Petroleum Geology, vol. 21, no. 10, pp. 1255-1264, 2004.

[107] L. Naudts, J. Greinert, Y. Artemov, S. E. Beaubien, C. Borowski, and M. D. Batist, "Anomalous sea-floor backscatter patterns in methane venting areas, Dnepr paleo-delta, NW Black Sea," Marine Geology, vol. 251, no. 3-4, pp. 253-267, 2008.

[108] M. Ergün, D. Dondurur, and G. Cifçi, “Acoustic evidence for shallow gas accumulations in the sediments of the Eastern Black Sea," Terra Nova, vol. 14, pp. 313-320, 2002.

[109] S. M. Karisiddaiah and M. Veerayya, "Occurrence of pockmarks and gas seepages along the central western continental margin of India," Current Science, vol. 82, pp. 52-57, 2002.

[110] N. Rollet, G. A. Logan, J. M. Kennard, P. E. O'Brien, A. T. Jones, and M. Sexton, "Characterisation and correlation of active hydrocarbon seepage using geophysical data sets: an example from the tropical, carbonate Yampi Shelf, Northwest Australia," Marine and Petroleum Geology, vol. 23, no. 2, pp. 145-164, 2006.

[111] B. J. Huang, X. M. Xiao, X. S. Li, and D. S. Cai, "Spatial distribution and geochemistry of the nearshore gas seepages and their implications to natural gas migration in the Yinggehai Basin, offshore South China Sea," Marine and Petroleum Geology, vol. 26, pp. 928-935, 2009. 
[112] X. J. Wang, B. Liu, J. Qian et al., "Geophysical evidence for gas hydrate accumulation related to methane seepage in the Taixinan Basin, South China Sea," Journal of Asian Earth Sciences, vol. 168, pp. 27-37, 2018.

[113] Y. T. Lu, X. W. Luan, F. L. Lyu et al., "Seismic evidence and formation mechanism of gas hydrates in the Zhongjiannan Basin, western margin of the South China Sea," Marine and Petroleum Geology, vol. 84, pp. 274-288, 2017.

[114] Y. N. Deng, F. Chen, Y. Hu et al., "Methane seepage patterns during the middle Pleistocene inferred from molybdenum enrichments of seep carbonates in the South China Sea," Ore Geology Reviews, vol. 125, p. 103701, 2020.

[115] D. G. Masson, B. J. Bett, D. S. M. Billett, C. L. Jacobs, A. J. Wheeler, and R. B. Wynn, "The origin of deep-water, coraltopped mounds in the northern Rockall Trough, Northeast Atlantic," Marine Geology, vol. 194, no. 3-4, pp. 159-180, 2003.

[116] W. C. Thomson, The Depths of the Sea, 13, MacMillan, London, 1874.

[117] S. Cairns, "Deep-water corals: an overview with special reference to diversity and distribution of deep-water Scleractinia," Bulletin of Marine Science, vol. 81, pp. 311-322, 2007.

[118] M. Hovland and E. Thomsen, "Cold-water corals-are they hydrocarbon seep related?," Marine Geology, vol. 137, no. 1-2, pp. 159-164, 1997.

[119] M. Hovland and E. Thomsen, "Hydrocarbon-based communities in the North Sea?," Sarsia, vol. 74, pp. 29-42, 1989.

[120] Y. Deng, F. Chen, N. Li, M. Jin, and S. Cheng, "Cold-water corals in gas hydrate drilling cores from the South China Sea: occurrences, geochemical characteristics and their relationship to methane seepages," Minerals, vol. 9, no. 12, p. 742, 2019.

[121] M. Taviani, A. Freiwald, and H. Zibrowius, "Deep coral growth in the Mediterranean Sea: an overview," in ColdWater Corals and Ecosystems. Erlangen Earth Conference Series, A. Freiwald and J. M. Roberts, Eds., Springer, Berlin Heidelberg, 2005.

[122] A. Carranza, A. M. Recio, M. Kitahara et al., "Deep-water coral reefs from the Uruguayan outer shelf and slope," Marine Biodiversity, vol. 42, no. 3, pp. 411-414, 2012.

[123] C. Van der Land, M. Eisele, and F. Mienis, "Carbonate mound development in contrasting settings on the Irish margin," Deep-Sea Research II, vol. 99, pp. 297-326, 2014.

[124] J. G. Colman, D. M. Gordon, A. P. Lane, M. J. Forde, and J. J. Fitzpatrick, "Carbonate mounds off Mauritania, Northwest Africa: status of deep-water corals and implications for management of fishing and oil exploration activities," in Cold-water corals and ecosystems, A. Freiwald and J. M. Roberts, Eds., pp. 417-441, Springer, Heidelberg, 2005.

[125] M. Eisele, N. Frank, C. Wienberg et al., "Productivity controlled cold-water coral growth periods during the last glacial off Mauritania," Marine Geology, vol. 280, no. 1-4, pp. 143149, 2011.

[126] E. K. L. Åström, M. L. Carroll, W. G. Ambrose Jr., and J. Carroll, "Arctic cold seeps in marine methane hydrate environments: impacts on shelf macrobenthic community structure offshore Svalbard," Marine Ecology Progress, vol. 552, pp. 1-18, 2016.

[127] The Porcupine-Belgica 97 Shipboard Party, J. P. Henriet, B. de Mol et al., "Gas hydrate crystals may help build reefs," Nature, vol. 391, no. 6668, pp. 648-649, 1998.
[128] D. Feng and D. F. Chen, "Authigenic carbonates from an active cold seep of the northern South China Sea: new insights into fluid sources and past seepage activity," DeepSea Research II, vol. 122, pp. 74-83, 2015.

[129] A. V. Milkov, "Worldwide distribution of submarine mud volcanoes and associated gas hydrates," Marine Geology, vol. 167, no. 1-2, pp. 29-42, 2000.

[130] C. Hensen, M. Nuzzo, E. Hornibrook et al., "Sources of mud volcano fluids in the Gulf of Cadiz-indications for hydrothermal imprint," Geochimica et Cosmochimica Acta, vol. 71, no. 5, pp. 1232-1248, 2007.

[131] A. J. Kopf, “Signigicance of mud volcanism," Reviews of Geophysics, vol. 40, no. 2, 2002.

[132] M. Hovland, A. Holl, and H. D. Stokes, "The structure and geomorphology of the Dashgil mud volcano, Azerbaijan," Geomorphology, vol. 21, pp. 1-15, 1997.

[133] A. Mazzini, A. Nermoen, M. Krotkiewski, Y. Podladchikov, S. Planke, and H. Svensen, "Strike-slip faulting as a trigger mechanism for overpressure release through piercement structures. Implications for the Lusi mud volcano, Indonesia," Marine \& Petroleum Geology, vol. 26, no. 9, pp. 1751$1765,2009$.

[134] G. D. Ginsburg and V. A. Solovie, "Mud volcano gas hydrates in the Caspian Sea," Bulletin of the Geological Society of Denmark, vol. 41, no. 6, pp. 95-100, 1994.

[135] L. L. Mazurenko, V. A. Soloviev, I. Belenkaya, M. K. Ivanov, and L. M. Pinheiro, "Mud volcano gas hydrates in the Gulf of Cadiz,” Terra Nova, vol. 14, no. 5, pp. 321-329, 2002.

[136] P. Van Rensbergen, M. de Batist, J. Klerkx et al., "Sublacustrine mud volcanoes and methane seeps caused by dissociation of gas hydrates in Lake Baikal," Geology, vol. 30, no. 7, pp. 631-634, 2002.

[137] V. H. Magalhaes, B. Buffett, D. Archer, P. C. McGuire, L. M. Pinheiro, and J. M. Gardner, "Effects of oceanographic changes on controlling the stability of gas hydrates and the formation of authigenic carbonates at mud volcanoes and seepage sites on the Iberian margin of the Gulf of Cadiz," Marine Geology, vol. 412, no. 69-80, pp. 69-80, 2019.

[138] M. Hovland and H. Svensen, "Submarine pingoes: indicators of shallow gas hydrates in a pockmark at Nyegga, Norwegian Sea," Marine Geology, vol. 228, no. 1-4, pp. 15-23, 2006.

[139] C. Serié, M. Huuse, and N. H. Schodt, "Gas hydrate pingoes: deep seafloor evidence of focused fluid flow on continental margins," Geology, vol. 40, no. 3, pp. 207-210, 2012.

[140] M. Hovland, "Geomorphological, geophysical, and geochemical evidence of fluid flow through the seabed," Journal of Geochemical Exploration, vol. 78, no. 3, pp. 287-291, 2003.

[141] V. Liebetrau, A. Eisenhauer, and P. Linke, "Cold seep carbonates and associated cold-water corals at the Hikurangi Margin, New Zealand: new insights into fluid pathways, growth structures and geochronology," Marine Geology, vol. 272, pp. 307-318, 2010.

[142] A. T. Jones, J. Greinert, D. A. Bowden et al., "Acoustic and visual characterisation of methane-rich seabed seeps at Omakere Ridge on the Hikurangi Margin, New Zealand," Marine Geology, vol. 272, no. 1-4, pp. 154-169, 2010.

[143] R. León, L. Somoza, T. Medialdea et al., "Sea-floor features related to hydrocarbon seeps in deepwater carbonate-mud mounds of the Gulf of Cádiz: from mud flows to carbonate precipitates," Geo-Marine Letters, vol. 27, no. 2-4, pp. 237247, 2007. 
[144] W. W. Schroeder, "Observations of Lophelia pertusa and the surficial geology at a deep-water site in the northeastern Gulf of Mexico," Hydrobiologia, vol. 471, no. 1-3, pp. 29-33, 2002.

[145] P. B. Mortensen, T. Hovland, J. H. Foss, and D. M. Furevik, "Distribution, abundance and size of Lophelia pertusa coral reefs in mid-Norway in relation to seabed characteristics," Journal of the Marine Biological Association of the UK, vol. 81, no. 4, pp. 4978-4996, 2001.

[146] S. E. Gass and J. Willison, An Assessment of the Distribution of Deep-Sea Corals in Atlantic Canada by Using Both Scientific and Local Forms of Knowledge, Springer, Berlin Heidelberg, 2005.

[147] L. A. Henry and J. M. Roberts, "Biodiversity and ecological composition of macrobenthos on cold-water coral mounds and adjacent off-mound habitat in the bathyal Porcupine Seabight, NE Atlantic," Deep Sea Research Part I Oceanographic Research Papers, vol. 54, no. 4, pp. 654-672, 2007.

[148] J. K. Reed, D. C. Weaver, and S. A. Pomponi, "Habitat and fauna of deep-water Lophelia pertusa coral reefs off the southeastern U.S.: Blake Plateau, Straits of Florida, and Gulf of Mexico," Bulletin of Marine Science, vol. 78, no. 2, pp. 343-375, 2006.

[149] E. E. Cordes, M. P. McGinley, E. L. Podowski et al., "Coral communities of the deep Gulf of Mexico," Deep-sea Research I, vol. 55, no. 6, pp. 777-787, 2008.

[150] R. E. Thresher, B. Tilbrook, S. Fallon, N. C. Wilson, and J. Adkins, "Effects of chronic low carbonate saturation levels on the distribution, growth and skeletal chemistry of deepsea corals and other seamount megabenthos," Marine Ecology Progress, vol. 442, no. 5, pp. 87-99, 2011.

[151] D. M. Tracey, A. A. Rowden, K. A. Mackay, and T. Compton, "Habitat-forming cold-water corals show affinity for seamounts in the New Zealand region," Marine Ecology Progress, vol. 430, no. 8, pp. 1-22, 2011.

[152] V. A. Huvenne, P. A. Tyler, D. G. Masson et al., "A picture on the wall: innovative mapping reveals cold-water coral refuge in submarine canyon," PLOS ONE, vol. 6, no. 12, article 28755, 2011.

[153] L. de Mol, D. van Rooij, H. Pirlet et al., "Cold-water coral habitats in the Penmarc'h and Guilvinec Canyons (Bay of Biscay): deep-water versus shallow-water settings," Marine Geology, vol. 282, no. 1-2, pp. 40-52, 2011.

[154] P. B. Mortensen, L. Buhl-Mortensen, A. V. Gebruk, and E. M. Krylova, "Occurrence of deep-water corals on the MidAtlantic Ridge based on MAR-ECO data," Deep Sea Research Part II, vol. 55, no. 1-2, pp. 142-152, 2008.

[155] A. Freiwald, L. Beuckm, A. Rüggeberg, M. Taviani, and D. Hebbenin, "The white coral community in the central Mediterranean Sea revealed by ROV surveys," Oceanography, vol. 22, no. 1, pp. 58-74, 2009.

[156] B. de Mol, P. van Rensbergen, S. Pillen et al., "Large deepwater coral banks in the Porcupine Basin, southwest of Ireland," Marine Geology, vol. 188, no. 1-2, pp. 193-231, 2002.

[157] N. H. Kenyon, A. M. Akhmetzhanov, A. J. Wheeler, T. C. E. V. Weering, and M. K. Ivanov, "Giant carbonate mud mounds in the southern Rockall Trough," Marine Geology, vol. 195, no. 1-4, pp. 5-30, 2003.

[158] M. Hovland, Deep-Water Coral Reefs, Springer, Netherlands, 2008.
[159] G. Wefer, D. Billet, D. Hebbeln, B. B. Jorgensen, M. Schlüter, and T. C. E. Van Weering, Ocean Margin Systems, Springer, Berlin, 2002.

[160] A. Gay, M. Lopez, C. Berndt, and M. Séranne, “Geological controls on focused fluid flow associated with seafloor seeps in the Lower Congo Basin," Marine Geology, vol. 244, pp. 68-92, 2007.

[161] S. Gullapalli, P. Dewangan, A. Kumar, G. Dakara, and C. K. Mishra, "Seismic evidence of free gas migration through the gas hydrate stability zone (GHSZ) and active methane seep in Krishna-Godavari offshore basin," Marine and Petroleum Geology, vol. 110, pp. 695-705, 2019.

[162] S. Sarkar, C. Berndt, T. A. Minshull et al., "Seismic evidence for shallow gas-escape features associated with a retreating gas hydrate zone offshore west Svalbard," Journal of Geophysical Research Solid Earth, vol. 117, article 09102, 2012.

[163] A. Portnov, A. J. Smith, J. Mienert, G. Cherkashov, and B. Vanshtein, "Offshore permafrost decay and massive seabed methane escape in water depths $>20 \mathrm{~m}$ at the South Kara Sea shelf," Geophysical Research Letters, vol. 40, no. 15, pp. 3962-3967, 2013.

[164] S. Merey and S. N. Longinos, "Investigation of gas seepages in Thessaloniki mud volcano in the Mediterranean Sea," Journal of Petroleum Science \& Engineering, vol. 168, pp. 51-97, 2018.

[165] J. Idczak, A. Brodecka-Goluch, K. Łukawska-Matuszewska et al., "A geophysical, geochemical and microbiological study of a newly discovered pockmark with active gas seepage and submarine groundwater discharge (MET1-BH, central Gulf of Gdańsk, southern Baltic Sea)," Science of The Total Environment, vol. 10, no. 742, article 140306, 2020. 